\title{
NEW COUNTEREXAMPLES TO THE CELL FORMULA IN NONCONVEX HOMOGENIZATION
}

\author{
MARCO BARCHIESI \& ANTOINE GLORIA
}

\begin{abstract}
In this article we show that for the homogenization of multiple integrals, the quasiconvexification of the cell formula is different from the asymptotic formula in general. To this aim, we construct three examples in three different settings: the homogenization of a discrete model, the homogenization of a composite material and the homogenization of a homogeneous material on a perforated domain.
\end{abstract}

Keywords: homogenization, quasiconvexity, cell formula

2000 Mathematics Subject Classification: 35B27, 49J45, 73B27, 74E30, 74Q05.

\section{Contents}

1. Introduction 1

2. Homogenization of multiple integrals and the cell formula 3

2.1. Continuous and discrete homogenization of nonconvex functionals 3

2.2. Short summary of convexity properties 5

2.3. Stefan Müller's counterexample 9

2.4. Counterexample by comparison of the zero levelsets 11

3. Counterexamples from composite materials 11

3.1. Discrete example 12

3.2. An example from solid-solid phase transformations 14

3.3. Comparison of boths examples 18

4. Counterexample on perforated domains 18

Appendix: Stefan Müller's example in dimension three 25

References 29

\section{INTRODUCTION}

For the homogenization of periodic integral functionals of the type

$$
I_{\varepsilon}(u):=\int_{\Omega \cap \varepsilon P} W\left(\frac{x}{\varepsilon}, \nabla u(x)\right) d x,
$$

with suitable assumptions (recalled in Section 2.1), the $\Gamma$-limit writes

$$
I_{\text {hom }}(u):=\int_{\Omega} W_{\text {hom }}(\nabla u(x)) d x,
$$

where $W_{\text {hom }}$ is obtained by an asymptotic formula on the number of periodic cells considered. If the integrand $W(x, \cdot)$ happens to be convex almost everywhere, then the 
asymptotic formula reduces to a minimization problem on the unitary cell with periodic boundary conditions, that we denote by $W_{\text {cell }}$. A counterexample due to Stefan Müller in [12] shows that in general, for quasiconvex nonconvex energy densities, the inequality $W_{\text {cell }} \geq W_{\text {hom }}$ can be strict. More recently, Jean-François Babadjian and the first author gave another such example in [3].

As will be made precise in Section 2 for both examples, the energy density $W_{\text {cell }}$ is not rank-one convex. In addition, in both cases, considering the quasiconvex envelop $Q W_{\text {cell }}$ of $W_{\text {cell }}$ surprisingly removes the contradiction which allows to conclude that $W_{\text {cell }}>W_{\text {hom }}$. Hence, none of the known examples shows that the inequality $Q W_{\text {cell }} \geq W_{\text {hom }}$ can be strict, although this is to be expected.

The aim of this paper is twofold: to show that the known counterexamples to the cell formula are not rigid enough to prove that $Q W_{\text {cell }}>W_{\text {hom }}$, and then to provide some new examples for which the latter strict inequality can be shown. The article is organized as follows. In Section 2, we recall standard results on homogenization as well as the two counterexamples to the cell formula mentioned above. We then show for each example that the methods used by their respective authors to prove the disagreement of $W_{\text {cell }}$ with $W_{\text {hom }}$ fail to prove the disagreement of $Q W_{\text {cell }}$ with $W_{\text {hom }}$. The rest of the paper is then dedicated to the construction of three different examples for which there exists a deformation gradient $\Lambda$ such that $Q W_{\text {cell }}(\Lambda)>W_{\text {hom }}(\Lambda)$. The examples are built in dimension two and they are based on the fact that replacing $(0,1)^{2}$-periodicity by $(0,2)^{2}$-periodicity is enough to relax significantly the energy to obtain the desired strict inequality. The first example is a discrete example where the keyrole is played by the very strong rigidity of discrete gradients. The second example is based on the same geometry but is written in a continuous setting and exploits the rigidity of the incompatible twowell problem together with an interplay between the geometry, the periodicity and the zero levelset of the energy densities. These examples are presented in Section 3. The last example is the object of Section 4. It relies on the homogenization of a homogeneous material on a perforated domain, for which we prove that the zero levelset of $W_{\text {cell }}$ is contained in a quasiconvex set which is strictly contained in the zero levelset of $W_{\text {hom }}$. This is in particular the first example which shows the disagreement of $W_{\text {cell }}$ and $W_{\text {hom }}$ (as well as $Q W_{\text {cell }}$ and $W_{\text {hom }}$ ) for the homogenization of a homogeneous material on a perforated domain.

Although the main result of this article is technical, we believe the examples are of independent interest. We therefore provide the non-specialist reader with the required background on convexity properties in Section 2.2 .

Throughout the paper, we employ the following notation:

- $\Omega$ is a bounded open subset of $\mathbb{R}^{d}$;

- $Q=(0,1)^{d}$ denotes the unit cell;

- $Q_{n}=(0, n)^{d}$ for all $n \in \mathbb{N}$;

- $Q^{m}=m+Q$ for all $m \in \mathbb{Z}^{d}$;

- $\chi_{U}$ is the characteristic function of a subset $U$ of $\mathbb{R}^{d}$;

- $\mathbb{M}^{d}$ is the set of $d \times d$ real matrices;

- $\mathbb{M}_{\text {sym }}^{d}$ is the set of $d \times d$ symmetric real matrices;

- $S O_{d}$ is the family of the elements $\Lambda$ of $\mathbb{M}^{d}$ such that $\operatorname{det} \Lambda=1$ and $\Lambda^{T} \Lambda=\mathbb{I}$, where $\mathbb{I} \in \mathbb{M}^{d}$ is the identity matrix; 
- $|\Lambda|:=\sqrt{\operatorname{trace}\left(\Lambda^{T} \Lambda\right)}$ is the Frobenius norm of a matrix $\Lambda \in \mathbb{M}^{d}$;

- $\mathcal{L}^{n}$ denotes the $n$-dimensional Lebesgue measure;

- $W_{\text {per }}^{1, p}\left(Q_{n}, \mathbb{R}^{d}\right)$ is the space of $W_{\text {loc }}^{1, p}\left(\mathbb{R}^{d} ; \mathbb{R}^{d}\right)$ functions which are $Q_{n}$-periodic;

- As a general rule, $c$ denotes a constant which may vary from line to line but which is independent of the variables left.

\section{Homogenization of MUltiple integrals AND THE CELl FORMula}

2.1. Continuous and discrete homogenization of nonconvex functionals. In this section, we recall classical results of periodic homogenization of multiple integrals, as well as (less) classical results of periodic homogenization of discrete systems. We refer the reader to the monograph [7] for continuous homogenization and to the article [1] for discrete homogenization.

Definition 1. Let $\mathcal{U}$ be a normed space. We say that $I: \mathcal{U} \rightarrow[-\infty,+\infty]$ is the $\Gamma$-limit of a sequence $I_{h}: \mathcal{U} \rightarrow[-\infty,+\infty]$, or that $I_{h} \Gamma$-converges to $I$, if for every $u \in \mathcal{U}$ the following conditions are satisfied:

i) Liminf inequality: for every sequence $u_{h}$ in $\mathcal{U}$ such that $u_{h} \rightarrow u$,

$$
I(u) \leq \liminf _{h \rightarrow+\infty} I_{h}\left(u_{h}\right)
$$

ii) Recovery sequence: there exists a sequence $u_{h}$ in $\mathcal{U}$ such that $u_{h} \rightarrow u$ and

$$
I(u)=\lim _{h \rightarrow+\infty} I_{h}\left(u_{h}\right) .
$$

Let $d \in \mathbb{N}$. We focus on $\Gamma$-convergence of integral functionals on the normed space $L^{p}\left(\Omega, \mathbb{R}^{d}\right), p \in(1,+\infty)$, in the context of periodic homogenization.

Let $a>0$. We denote by $\mathcal{W}(a, p)$ the set of all continuous functions $W: \mathbb{M}^{d} \rightarrow[0,+\infty)$ satisfying the following coerciveness and growth conditions of order $p$ :

$$
\frac{1}{a}|\Lambda|^{p}-a \leq \mathcal{W}(\Lambda) \leq a\left(1+|\Lambda|^{p}\right) \quad \text { for all } \Lambda \in \mathbb{M}^{d} .
$$

Hypothesis 1 . $W: \mathbb{R}^{d} \times \mathbb{M}^{d} \rightarrow[0,+\infty)$ is a Carathéodory function $Q$-periodic in the first variable such that $W(x, \cdot) \in \mathcal{W}(a, p)$ for a.e $x \in Q$.

Hypothesis 2. $P$ is a $Q$-periodic and open subset of $\mathbb{R}^{d}$ with Lipschitz boundary such that $Q \backslash P \subset \subset Q$. Note that in particular $P$ is connected.

Under Hypotheses 1 and 2, we consider for any $\varepsilon>0$ the functional $I_{\varepsilon}: L^{p}\left(\Omega, \mathbb{R}^{d}\right) \rightarrow$ $[0,+\infty]$ defined by

$$
I_{\varepsilon}(u):= \begin{cases}\int_{\Omega \cap \varepsilon P} W\left(\frac{x}{\varepsilon}, \nabla u(x)\right) d x & \text { if }\left.u\right|_{\Omega \cap \varepsilon P} \in W^{1, p}\left(\Omega \cap \varepsilon P, \mathbb{R}^{d}\right), \\ +\infty & \text { otherwise. }\end{cases}
$$

Definition 2. We call cell integrand related to $(W, P)$ the function $W_{\text {cell }}: \mathbb{M}^{d} \rightarrow[0,+\infty)$ defined by

$$
W_{\text {cell }}(\Lambda):=\inf \left\{\int_{Q \cap P} W(x, \Lambda+\nabla \phi(x)) d x: \phi \in W_{\text {per }}^{1, p}\left(Q, \mathbb{R}^{d}\right)\right\} .
$$


If $P=\mathbb{R}^{d}$ we simply say that $W_{\text {cell }}$ is the cell integrand related to $W$.

We call homogenized integrand related to $(W, P)$ the function $W_{\text {hom }}: \mathbb{M}^{d} \rightarrow[0,+\infty)$ defined by

$$
W_{\text {hom }}(\Lambda):=\lim _{n \rightarrow \infty} \frac{1}{n^{d}} \inf \left\{\int_{Q_{n} \cap P} W(x, \Lambda+\nabla \phi(x)) d x: \phi \in W_{\text {per }}^{1, p}\left(Q_{n}, \mathbb{R}^{d}\right)\right\} .
$$

If $P=\mathbb{R}^{d}$ we simply say that $W_{\text {hom }}$ is the homogenized integrand related to $W$.

The following theorem is a standard result (See [7, Theorem 19.1 and Remark 19.2]).

Theorem 1. Assume that $W$ satisfies Hypothesis 1 and that $P$ satisfies Hypothesis 2. Then the homogenized integrand $W_{\text {hom }}$ related to $(W, P)$ is a quasiconvex function satisfying (2.1), and for any $\varepsilon_{h} \searrow 0^{+}$the sequence $I_{\varepsilon_{h}} \quad \Gamma$-converges to the functional $I_{\text {hom }}$ : $L^{p}\left(\Omega, \mathbb{R}^{d}\right) \rightarrow[0,+\infty]$ defined by

$$
I_{\mathrm{hom}}(u):= \begin{cases}\int_{\Omega} W_{\mathrm{hom}}(\nabla u(x)) d x & \text { if } u \in W^{1, p}\left(\Omega, \mathbb{R}^{d}\right) \\ +\infty & \text { otherwise. }\end{cases}
$$

In addition, if $W(x, \cdot)$ is convex for a.e. $x \in Q$, then $W_{\text {hom }}$ is also convex and coincides with the cell integrand $W_{\text {cell }}$ related to $(W, P)$.

A result similar to Theorem 1 holds in a discrete setting, as shown in [1]. We give here a simpler version, for which we only consider nearest-neighbors interactions. We also need to slightly extend the result in [1] to take into account volumetric effects, which we will need in Section 3. Yet, the result remains essentially the same and further details can be found in [2].

Definition 3. Let $\mathcal{T}$ be a $Q$-periodic triangulation of $\mathbb{R}^{d}$ and $\mathcal{P}$ be the set of vertices of $\mathcal{T}$. We define the couples of nearest neighbors by

$$
\mathcal{N N}:=\left\{(x, y) \in(\mathcal{P} \cap \bar{Q})^{2}: \exists T \in \mathcal{T} \text { having }[x, y] \text { as an edge }\right\} .
$$

For all $\varepsilon>0$ and for all bounded open subset $U$ of $\mathbb{R}^{d}$, we define

$$
\mathcal{S}_{\varepsilon}\left(U, \mathbb{R}^{d}\right):=\left\{u \in C^{0}\left(U, \mathbb{R}^{d}\right): u \text { is affine on each element } T \in \varepsilon \mathcal{T} \cap U\right\} .
$$

For $\varepsilon=1$, we simply write $\mathcal{S}\left(U, \mathbb{R}^{d}\right)=\mathcal{S}_{\varepsilon}\left(U, \mathbb{R}^{d}\right)$. Moreover, we write

$$
\mathcal{S}_{\text {per }}\left(Q_{n}, \mathbb{R}^{d}\right):=\mathcal{S}\left(Q_{n}, \mathbb{R}^{d}\right) \cap W_{\text {per }}^{1, \infty}\left(Q_{n}, \mathbb{R}^{d}\right) .
$$

We are now in position to define energy functionals on discrete systems.

Definition 4. Let $f_{1}: Q \times Q \times \mathbb{R}^{d} \rightarrow[0,+\infty)$ and $f_{2}: Q \times \mathbb{R} \rightarrow[0,+\infty)$ be continuous functions and let $\varepsilon>0$. For any bounded open subset $U$ of $\mathbb{R}^{d}$, we define the energy of $u \in \mathcal{S}_{\varepsilon}\left(U, \mathbb{R}^{d}\right)$ as

$$
F_{\varepsilon}(u, U):=\sum_{m \in \mathbb{Z}^{d}: \varepsilon Q^{m} \subseteq U} F_{\varepsilon}^{m}(u),
$$

where, for any $m \in \mathbb{Z}^{d}$ such that $\varepsilon Q^{m} \subseteq U$,

$$
F_{\varepsilon}^{m}(u):=\varepsilon^{d} \sum_{(x, y) \in \mathcal{N N}} f_{1}\left(x, y, \frac{u(\varepsilon m+\varepsilon x)-u(\varepsilon m+\varepsilon y)}{\varepsilon|x-y|}\right)+\varepsilon^{d} \int_{Q} f_{2}(x, \operatorname{det} \nabla u(\varepsilon m+\varepsilon x)) d x .
$$


If $\varepsilon=1$, we simply write $F(u, U)=F_{\varepsilon}(u, U)$. Finally, we define the functional $I_{\varepsilon}$ : $L^{p}\left(\Omega, \mathbb{R}^{d}\right) \rightarrow[0,+\infty]$ by

$$
I_{\varepsilon}(u):= \begin{cases}F_{\varepsilon}(u, \Omega) & \text { if } u \in S_{\varepsilon}\left(\Omega, \mathbb{R}^{d}\right), \\ +\infty & \text { otherwise. }\end{cases}
$$

As for the continuous setting, we may define a cell integrand and a homogenized integrand as follows.

Definition 5. For all $\Lambda \in \mathbb{M}^{d}$, let $\varphi_{\Lambda}: \mathbb{R}^{d} \rightarrow \mathbb{R}^{d}$ be given by $\varphi_{\Lambda}(x):=\Lambda \cdot x$. We call cell integrand related to $(\mathcal{T}, F)$ the function $W_{\text {cell }}: \mathbb{M}^{d} \rightarrow[0,+\infty)$ defined by

$$
W_{\text {cell }}(\Lambda):=\inf \left\{F\left(\varphi_{\Lambda}+\phi, Q\right): \phi \in \mathcal{S}_{\text {per }}\left(Q, \mathbb{R}^{d}\right)\right\} .
$$

We call homogenized integrand related to $(\mathcal{T}, F)$ the function $W_{\text {hom }}: \mathbb{M}^{d} \rightarrow[0,+\infty)$ defined by

$$
W_{\mathrm{hom}}(\Lambda):=\lim _{n \rightarrow \infty} \frac{1}{n^{d}} \inf \left\{F\left(\varphi_{\Lambda}+\phi, Q_{n}\right): \phi \in \mathcal{S}_{\mathrm{per}}\left(Q_{n}, \mathbb{R}^{d}\right)\right\}
$$

We have the following result (see [1] and [2]).

Theorem 2. Let $\mathcal{T}, f_{1}, f_{2}, I_{\varepsilon}$ and $W_{\text {hom }}$ be as in Definitions 3, 4, 5. Let us further assume that there exist $a>0$ and $p \in(1, \infty)$ such that

$$
\begin{aligned}
& 0 \leq f_{2}(x, z) \leq a\left(1+|z|^{p / d}\right) \text { for all }(x, z) \in Q \times \mathbb{R}, \\
& \frac{1}{a}|w|^{p}-a \leq f_{1}(x, y, w) \leq a\left(1+|w|^{p}\right) \text { for all }(x, y, w) \in Q \times Q \times \mathbb{R}^{d},
\end{aligned}
$$

Then the homogenized integrand $W_{\mathrm{hom}}$ associated to $(\mathcal{T}, F)$ is a quasiconvex function satisfying a growth condition (2.1), and for any $\varepsilon_{h} \searrow 0^{+}$the sequence $I_{\varepsilon_{h}} \Gamma$-converges to the functional $I_{\mathrm{hom}}: L^{p}\left(\Omega, \mathbb{R}^{d}\right) \rightarrow[0,+\infty]$ defined by

$$
I_{\mathrm{hom}}(u):= \begin{cases}\int_{\Omega} W_{\mathrm{hom}}(\nabla u(x)) d x & \text { if } u \in W^{1, p}\left(\Omega, \mathbb{R}^{d}\right), \\ +\infty & \text { otherwise. }\end{cases}
$$

In addition, if $f_{2} \equiv 0$ and if $f_{1}(x, y, \cdot)$ is a convex function for all $x, y \in Q$, then $W_{\text {hom }}$ is also convex and coincides with the cell integrand $W_{\text {cell }}$ related to $(\mathcal{T}, F)$.

2.2. Short summary of convexity properties. In this section, we recall the notions of polyconvexity, quasiconvexity and rank-one convexity of functions and sets. We refer the reader to $[8,9,13]$ for details. We also state and prove some elementary lemmas that will be used in the analysis of the counterexamples.

Definition 6. (quasiconvex function) Let $W: \mathbb{M}^{d} \rightarrow \mathbb{R}$ be locally bounded and Borel measurable. Its quasiconvex envelope $Q W: \mathbb{M}^{d} \rightarrow[-\infty,+\infty)$ is defined by

$$
Q W(\Lambda):=\inf \left\{f_{U} W(\Lambda+\nabla \phi(x)) d x: \phi \in W_{0}^{1, \infty}\left(U, \mathbb{R}^{d}\right)\right\},
$$

where $U$ is a bounded open subset of $\mathbb{R}^{d}$. In particular, the infimum in the formula is independent of the choice of $U$. If $U=Q$, then $W_{0}^{1, \infty}\left(Q, \mathbb{R}^{d}\right)$ can be replaced by 
$W_{\text {per }}^{1, \infty}\left(Q, \mathbb{R}^{d}\right)$. The function $W$ is said to be quasiconvex if $W=Q W$. If $Q W$ is finite, then it is quasiconvex.

Lemma 1. (main property) Let $W: \Omega \times \mathbb{M}^{d} \rightarrow[0,+\infty)$ be a Carathéodory function such that $W(x, \cdot) \in \mathcal{W}(a, p)$ for a.e. $x \in \Omega$ and let $\mathcal{U}$ be a weakly closed subset of $W^{1, p}\left(\Omega, \mathbb{R}^{d}\right)$. Then

$$
\inf \left\{\int_{\Omega} W(x, \nabla u(x)) d x: u \in \mathcal{U}\right\}=\min \left\{\int_{\Omega} Q W(x, \nabla u(x)) d x: u \in \mathcal{U}\right\}>-\infty,
$$

and any weak limit of a minimizing sequence of the original problem is a minimizer of the relaxed problem.

Remark 1. Assume that $P$ satisfies Hypothesis 2. Since $Q \cap P$ has Lipschitz boundary, any function $\varphi \in W^{1, p}\left(Q \cap P, \mathbb{R}^{d}\right)$ can be extended to a function $\widetilde{\varphi} \in W^{1, p}\left(Q, \mathbb{R}^{d}\right)$. As a consequence, $\left\{\phi_{\mid Q \cap P}: \phi \in W_{\text {per }}^{1, p}\left(Q, \mathbb{R}^{d}\right)\right\}$ is a weakly closed subset of $W^{1, p}\left(Q \cap P, \mathbb{R}^{d}\right)$.

Definition 7. (polyconvex function) For any matrix $\Lambda \in \mathbb{M}^{d}$, let denote by $\mathbf{M}(\Lambda)$ the vector that consists of all minors of $\Lambda$, and denote by $\delta(d)$ its length. We can identify $\mathbf{M}(\Lambda)$ with a point of $\mathbb{R}^{\delta(d)}$. We say that a function $W: \mathbb{M}^{d} \rightarrow \mathbb{R}$ is polyconvex if there exists a convex function $g: \mathbb{R}^{\delta(d)} \rightarrow \mathbb{R}$ such that for all $\Lambda \in \mathbb{M}^{d}$,

$$
W(\Lambda)=g(\mathbf{M}(\Lambda)) \text {. }
$$

Definition 8. (rank-one convex function) We say that $W: \mathbb{M}^{d} \rightarrow \mathbb{R}$ is rank-one convex if

$$
W(t A+(1-t) B) \leq t W(A)+(1-t) W(B)
$$

for all $t \in[0,1]$ and for all $A, B \in \mathbb{M}^{d}$ rank-one connected, i.e., such that $\operatorname{rank}(B-A)=1$.

Lemma 2. Let $W: \mathbb{M}^{d} \rightarrow \mathbb{R}$, then there holds

$W$ is convex $\Longrightarrow W$ is polyconvex $\Longrightarrow W$ is quasiconvex $\Longrightarrow W$ is rank-one convex.

One can extend the notions of convexity, polyconvexity, quasiconvexity and rank-one convexity to sets.

Definition 9. (polyconvex, quasiconvex and rank-one convex sets) Let $K$ be a compact subset of $\mathbb{M}^{d}$. We define the polyconvex hull $K^{p c}$, quasiconvex hull $K^{q c}$ and rank-one convex hull $K^{r c}$ of $K$ by

$$
\begin{aligned}
& K^{p c}:=\left\{\Lambda \in \mathbb{M}^{d}: f(\Lambda)=0 \quad \forall f: \mathbb{M}^{d} \rightarrow[0,+\infty) \text { polyconvex such that }\left.f\right|_{K} \equiv 0\right\}, \\
& K^{q c}:=\left\{\Lambda \in \mathbb{M}^{d}: f(\Lambda)=0 \quad \forall f: \mathbb{M}^{d} \rightarrow[0,+\infty) \text { quasiconvex such that }\left.f\right|_{K} \equiv 0\right\}, \\
& K^{r c}:=\left\{\Lambda \in \mathbb{M}^{d}: f(\Lambda)=0 \quad \forall f: \mathbb{M}^{d} \rightarrow[0,+\infty) \text { rank-one convex such that }\left.f\right|_{K} \equiv 0\right\} .
\end{aligned}
$$

The set $K$ is said to be polyconvex if $K=K^{p c}$, quasiconvex if $K=K^{q c}$ and rankone convex if $K=K^{r c}$. We have the inclusions $K^{r c} \subseteq K^{q c} \subseteq K^{p c} \subseteq K^{c o}$, where the superscript co denotes the classical convex hull.

We have the following useful characterizations of $K^{q c}$ and $K^{p c}$.

Lemma 3. ([13, Theorem 4.10]). Given a compact set $K \subseteq \mathbb{M}^{d}$, a matrix $A \in \mathbb{M}^{d}$ belongs to $K^{q c}$ if and only if there exists a sequence $\psi_{h}$ bounded in $W^{1, \infty}\left(Q, \mathbb{R}^{d}\right)$ such that

$$
\begin{aligned}
& \operatorname{dist}\left(\nabla \psi_{h}, K\right) \rightarrow 0 \text { in measure; } \\
& \psi_{h}(x)=A \cdot x \text { for } x \in \partial Q .
\end{aligned}
$$


Lemma 4. ([11, Lemma 1]). Given a compact set $K \subseteq \mathbb{M}^{d}$, a matrix $A \in \mathbb{M}^{d}$ belongs to $K^{p c}$ if and only if $\mathbf{M}(A)$ lies in $\{\mathbf{M}(\Lambda): \Lambda \in K\}^{c o}$.

The sets we will be interested in are the zero-levelsets of energy densities defined as follows.

Definition 10. Let $W: \mathbb{M}^{d} \rightarrow[0,+\infty)$ be a continuous function, we define its zero levelset as

$$
W^{-1}(0)=\left\{\Lambda \in \mathbb{M}^{d}: W(\Lambda)=0\right\} .
$$

In particular, if $W$ is a quasiconvex function, then $W^{-1}(0)$ is a quasiconvex set.

Lemma 5. If $W \in \mathcal{W}(a, p)$, then

$$
Q W^{-1}(0)=\left(W^{-1}(0)\right)^{q c} .
$$

Proof. Let $K:=W^{-1}(0)$. The inclusion $K^{q c} \subseteq Q W^{-1}(0)$ is trivial and we only need to prove the opposite one. This proof makes use of Young measures, for which we refer the reader to [13] for a comprehensive treatment.

Let $A \in Q W^{-1}(0)$. By definition of the quasiconvex envelope, there exists $\phi_{h} \in$ $W_{0}^{1, \infty}\left(Q, \mathbb{R}^{d}\right)$ such that

$$
0=Q W(A)=\lim _{h \rightarrow+\infty} \int_{Q} W\left(A+\nabla \phi_{h}(x)\right) d x .
$$

As a consequence of the $p$-coercivity of $W$ and Poincaré's inequality, the sequence $\psi_{h}(x):=$ $A \cdot x+\phi_{h}(x)$ is bounded in $W^{1, p}\left(Q, \mathbb{R}^{d}\right)$. Thus, up to extraction, $\nabla \psi_{h}$ generates a Young measure $\mu: Q \ni x \mapsto \mu_{x} \in \mathcal{P}\left(\mathbb{M}^{d}\right)$, where $\mathcal{P}\left(\mathbb{M}^{d}\right)$ denotes the family of probability measures on $\mathbb{M}^{d}$.

By the fundamental theorem on Young measures (see [13, Theorem 3.1]), we get

$$
0=\lim _{h \rightarrow+\infty} \int_{Q} W\left(A+\nabla \phi_{h}(x)\right) d x \geq \int_{Q}\left(\int_{\mathbb{M}^{d}} W(\Lambda) d \mu_{x}(\Lambda)\right) d x
$$

and therefore by [5, Lemma 3.3] $\operatorname{supp} \mu_{x} \subseteq K$ for $\mathcal{L}^{d}$ a.e. $x \in Q$. Again by the fundamental theorem, this implies that

$$
\operatorname{dist}\left(\nabla \psi_{h}, K\right) \rightarrow 0 \text { in measure. }
$$

By using Zhang's lemma (see [13, Lemma 4.21]), $\psi_{h}$ can be modified on small sets so that its gradient be bounded in $L^{\infty}\left(Q, \mathbb{M}^{d}\right)$, while keeping conditions $(2.3)$ and $\psi_{h}(x)=A \cdot x$ for $x \in \partial Q$. The thesis follows now by Lemma 3 .

We will also make use of the following results about the cell integrand.

Lemma 6. Assume that $W$ satisfies Hypothesis 1 and that $P$ satisfies Hypothesis 2. Then the cell integrand $W_{\text {cell }}$ related to $(W, P)$ is a continuous function.

Proof. This property is a direct consequence of the following inequality:

$$
W_{\text {cell }}\left(\Lambda_{1}\right) \leq W_{\text {cell }}\left(\Lambda_{2}\right)+c\left(\Lambda_{1}, \Lambda_{2}\right)\left|\Lambda_{1}-\Lambda_{2}\right| \text { for all } \Lambda_{1}, \Lambda_{2} \in \mathbb{M}^{d},
$$

where $c\left(\Lambda_{1}, \Lambda_{2}\right)$ is locally uniformly bounded. 
Let us prove inequality (2.4). By Lemma 1 and Remark 1, we have

$$
W_{\text {cell }}(\Lambda)=\min \left\{\int_{Q \cap P} Q W(x, \Lambda+\nabla \phi(x)) d x: \phi \in W_{\text {per }}^{1, p}\left(Q, \mathbb{R}^{d}\right)\right\} .
$$

Due to the growth condition from above satisfied by $Q W(x, \cdot)$, there exists $c>0$ such that for every $\Lambda_{1}, \Lambda_{2} \in \mathbb{M}^{d}$, there holds

$$
\left|Q W\left(x, \Lambda_{1}\right)-Q W\left(x, \Lambda_{2}\right)\right| \leq c\left|\Lambda_{1}-\Lambda_{2}\right|\left(1+\left|\Lambda_{1}\right|^{p-1}+\left|\Lambda_{2}\right|^{p-1}\right)
$$

This property, which is classical for convex functions, holds for rank-one convex functions (see [10, Lemma 5.2]). Let now $\Lambda_{1}, \Lambda_{2} \in \mathbb{M}^{d}$, and let $\phi_{1}, \phi_{2} \in W_{\text {per }}^{1, p}\left(Q, \mathbb{R}^{d}\right)$ be minimizers associated with $\Lambda_{1}$ and $\Lambda_{2}$ through (2.5). We then have

$$
\begin{aligned}
W_{\text {cell }}\left(\Lambda_{1}\right)-W_{\text {cell }}\left(\Lambda_{2}\right) & =\int_{Q \cap P} Q W\left(x, \Lambda_{1}+\nabla \phi_{1}(x)\right)-Q W\left(x, \Lambda_{2}+\nabla \phi_{2}(x)\right) d x \\
& \leq \int_{Q \cap P} Q W\left(x, \Lambda_{1}+\nabla \phi_{2}(x)\right)-Q W\left(x, \Lambda_{2}+\nabla \phi_{2}(x)\right) d x \\
& \leq \int_{Q \cap P} c\left|\Lambda_{1}-\Lambda_{2}\right|\left(1+\left|\Lambda_{1}\right|^{p-1}+\left|\Lambda_{2}\right|^{p-1}+\left|\Lambda_{2}+\nabla \phi_{2}(x)\right|^{p-1}\right) d x .
\end{aligned}
$$

Using the coercivity of $Q W$ (lower bound in (2.1)), we may bound $\left\|\Lambda_{2}+\nabla \phi_{2}\right\|_{L^{p}}^{p}$ from above by the energy, which is less than $c\left(1+\left|\Lambda_{2}\right|^{p}\right)$ using the test function $\phi \equiv 0$ and the upper bound of (2.1). Hence, there exists a constant $c>0$ such that the inequality

$$
W_{\text {cell }}\left(\Lambda_{1}\right)-W_{\text {cell }}\left(\Lambda_{2}\right) \leq c\left|\Lambda_{1}-\Lambda_{2}\right|\left(1+\left|\Lambda_{1}\right|^{p-1}+\left|\Lambda_{2}\right|^{p-1}\right),
$$

holds for any $\Lambda_{1}, \Lambda_{2} \in \mathbb{M}^{d}$, which proves the claim.

Lemma 7. Let $W \in \mathcal{W}(a, p)$ and let $P$ satisfy Hypothesis 2. Assume in addition that $W$ is quasiconvex, and that $W^{-1}(0)$ is not empty. Then $A \in \mathbb{M}^{d}$ belongs to the zero levelset of the cell integrand $W_{\text {cell }}$ associated to $(W, P)$ if and only if there exists a $Q$-periodic Lipschitz function $\phi: \mathbb{R}^{d} \rightarrow \mathbb{R}^{d}$ satisfying

$$
A+\nabla \phi(x) \in W^{-1}(0) \quad \text { for a.e. } x \in Q \cap P .
$$

Proof. The condition is obviously sufficient. By Lemma 1 and Remark 1, we have

$$
W_{\text {cell }}(\Lambda)=\min \left\{\int_{Q \cap P} W(\Lambda+\nabla \phi(x)) d x: \phi \in W_{\text {per }}^{1, p}\left(Q, \mathbb{R}^{d}\right)\right\} .
$$

Let $A \in W_{\text {cell }}^{-1}(0)$ and let $\phi \in W_{\text {per }}^{1, p}\left(Q, \mathbb{R}^{d}\right)$ be a minimizer associated with $A$ through (2.6). Then

$$
W(A+\nabla \phi(x))=0 \text { for a.e. } x \in Q \cap P .
$$

Since $W^{-1}(0)$ is compact and $Q \cap P$ has a Lipschitz boundary, the function $\phi_{\mid Q \cap P}$ has a Lipschitz representative. The conclusion follows by taking a Lipschitz $Q$-periodic extension of $\phi_{\mid Q \cap P}$ on $\mathbb{R}^{d}$.

A similar characterization of the levelset of the cell integrand holds in the case of mixtures. 
Lemma 8. ([3, Lemma 4.4]). Let $W_{1}, W_{2} \in \mathcal{W}(a, p)$ be two quasiconvex functions such that $W_{1}^{-1}(0)$ and $W_{2}^{-1}(0)$ are not empty. Given a measurable subset $U$ of $Q$, let set $W: \mathbb{R}^{d} \times \mathbb{M}^{d} \rightarrow[0,+\infty)$ as

$$
W(x, \Lambda):=\chi(x) W_{1}(\Lambda)+(1-\chi(x)) W_{2}(\Lambda),
$$

where $\chi$ is defined by $\chi:=\chi_{U}$ in $Q$ and extended by periodicity to the whole $\mathbb{R}^{d}$. Then $A \in \mathbb{M}^{d}$ belongs to the zero levelset of the cell integrand $W_{\text {cell }}$ associated to $W$ if and only if there exists a $Q$-periodic Lipschitz function $\phi: \mathbb{R}^{d} \rightarrow \mathbb{R}^{d}$ satisfying

$$
A+\nabla \phi(x) \in\left\{\begin{array}{lll}
W_{1}^{-1}(0) & \text { for a.e. } & x \in U \\
W_{2}^{-1}(0) & \text { for a.e. } & x \in Q \backslash U
\end{array} .\right.
$$

Remark 2. The previous lemma shows that in the case of a mixture of the type $W=$ $\chi W_{1}+(1-\chi) W_{2}$, the zero levelset of $W_{\text {cell }}$ depends only on the zero levelsets of $W_{1}, W_{2}$ and not on their global shapes or growths. The same property can be proved for the zero levelset of $W_{\text {hom }}$ (see [6, Theorem 1.3]). This fact is one of the keys of our counterexamples: we have to introduce suitable zero levelsets first, and only afterwards construct suitable functions.

2.3. Stefan Müller's counterexample. The energy under consideration $W^{\eta}: \mathbb{R}^{2} \times$ $\mathbb{M}^{2} \rightarrow[0,+\infty),(x, \Lambda) \mapsto \chi^{\eta}(x) W_{0}(\Lambda)$ models a two-dimensional laminate composite, made of a strong material and a soft material. The coefficient $\chi^{\eta}$ is the $Q$-periodic extension on $\mathbb{R}^{2}$ of

$$
\chi^{\eta}(x):=\left\{\begin{array}{ll}
1 & \text { if } x_{1} \in(0,1 / 2) \\
\eta & \text { if } x_{1} \in[1 / 2,1)
\end{array},\right.
$$

where $Q \ni x=\left(x_{1}, x_{2}\right)$ and $\eta>0$. The energy density $W_{0}: \mathbb{M}^{2} \rightarrow[0,+\infty)$ is given by $W_{0}(\Lambda)=|\Lambda|^{4}+f(\operatorname{det} \Lambda)$ where

$$
f(z):= \begin{cases}\frac{8(1+a)^{2}}{z+a}-8(1+a)-4 & \text { if } z>0 \\ \frac{8(1+a)^{2}}{a}-8(1+a)-4-\frac{8(1+a)^{2}}{a^{2}} z & \text { if } z \leq 0\end{cases}
$$

for some $a \in(0,1 / 2)$.

In particular, $W^{\eta}(x, \cdot)$ is a nonnegative polyconvex function satisfying a standard growth condition (2.1) of order $p=4$. Its zero levelset is $S_{2}$ for all $x \in Q$.

We respectively denote by $W_{\text {cell }}^{\eta}$ and $W_{\text {hom }}^{\eta}$ the cell integrand and the homogenized integrand associated with $W^{\eta}$.

Using the one-well rigidity (Liouville theorem) on the unitary cell and using 'bucklinglike' test-functions on several periodic cells (see Figure 2.3), Stefan Müller obtained the following result.

Theorem 3. [12, Theorem 4.3] For all $\lambda \in(\pi / 4,1)$, there exist $c_{1}, c_{2}>0$ independent of $\eta$, such that

$$
\begin{aligned}
& W_{\text {hom }}^{\eta}(\Lambda) \leq \eta c_{1} \\
& W_{\text {cell }}^{\eta}(\Lambda) \geq c_{2},
\end{aligned}
$$



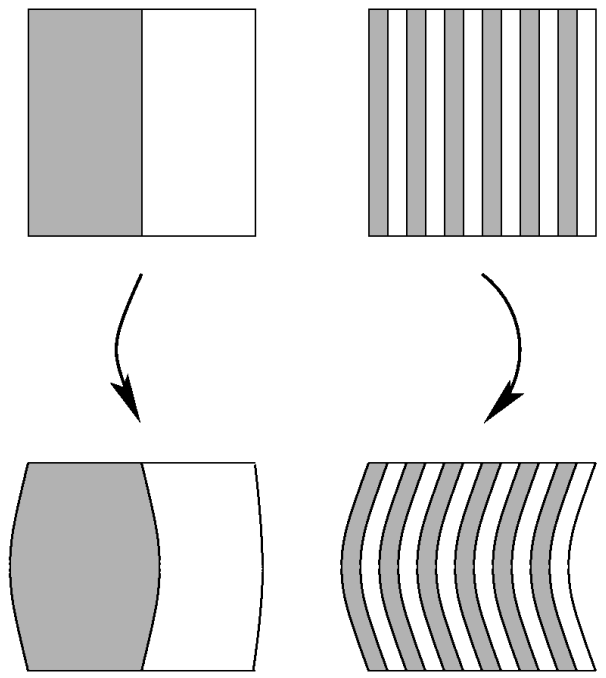

FiguRe 1. Compression of one periodic cell and buckling of several periodic cells

where $\Lambda:=\operatorname{diag}(1, \lambda)$, hence proving that the strict inequality $W_{\text {cell }}^{\eta}(\Lambda)>W_{\mathrm{hom}}^{\eta}(\Lambda)$ holds provided $\eta$ is small enough.

More precisely, it turns out that $W_{\text {cell }}^{\eta}$ is not even a quasiconvex function, as shown by the following proposition.

Proposition 1. For all $\lambda \in(0,1)$, there exists $c>0$ independent of $\eta$ such that

$$
Q W_{\text {cell }}^{\eta}(\Lambda) \leq \eta c
$$

where $\Lambda:=\operatorname{diag}(1, \lambda)$.

Hence, in view of Proposition 1, Theorem 3 does not allow to conclude whether the inequality $Q W_{\text {cell }}^{\eta}(\Lambda) \geq W_{\text {hom }}^{\eta}(\Lambda)$ may be strict or not.

Proof of Proposition 1. Since

$$
Q W_{\text {cell }}^{\eta}(\Lambda)=\inf \left\{\int_{Q} W_{\text {cell }}^{\eta}(\Lambda+\nabla \phi(x)) d x: \phi \in W_{\text {per }}^{1, \infty}\left(Q, \mathbb{R}^{2}\right)\right\}
$$

it is enough to exhibit a test function $\phi \in W_{\text {per }}^{1, \infty}\left(Q, \mathbb{R}^{2}\right)$ such that the majoration in (2.7) holds. Let $\phi \in W_{\text {per }}^{1, \infty}\left(Q, \mathbb{R}^{2}\right)$ be such that

$$
\nabla \phi(x)=\bar{\chi}(x)\left(\begin{array}{cc}
0 & \sqrt{1-\lambda^{2}} \\
0 & 0
\end{array}\right),
$$

where $\bar{\chi}$ is the $Q$-periodic extension on $\mathbb{R}^{2}$ of

$$
\bar{\chi}(x):=\left\{\begin{array}{ll}
1 & \text { if } x_{1} \in(0,1 / 2) \\
-1 & \text { if } x_{1} \in[1 / 2,1)
\end{array} .\right.
$$

We also choose $\varphi \in L^{\infty}\left(Q, W_{\text {per }}^{1, \infty}\left(Q, \mathbb{R}^{2}\right)\right)$ such that

$$
\nabla_{y} \varphi(x, y)=\bar{\chi}(y)\left(\begin{array}{cc}
\lambda-1 & 0 \\
\bar{\chi}(x) \sqrt{1-\lambda^{2}} & 0
\end{array}\right),
$$


Then, in the strong phase $(\bar{\chi}(y)=1)$, the test function $\Lambda+\nabla \phi(x)+\nabla_{y} \varphi(x, y)$ is the rotation

$$
\left(\begin{array}{cc}
\lambda & \pm \sqrt{1-\lambda^{2}} \\
\mp \sqrt{1-\lambda^{2}} & \lambda
\end{array}\right)
$$

and, in the soft phase $(\bar{\chi}(y)=-1)$, the deformation gradient is of the form

$$
A:=\left(\begin{array}{cc}
2-\lambda & \pm \sqrt{1-\lambda^{2}} \\
\pm \sqrt{1-\lambda^{2}} & \lambda
\end{array}\right) .
$$

Hence,

$$
Q W_{\text {cell }}^{\eta}(\Lambda) \leq \int_{Q} \int_{Q} W^{\eta}\left(y, \Lambda+\nabla \phi(x)+\nabla_{y} \varphi(x, y)\right) d y d x \leq \frac{1}{2} \eta W_{0}(A),
$$

for some $A$ of the form (2.9).

Remark 3. Since (2.8) is a rank-one matrix almost everywhere, the same proof shows that the bound in Proposition 1 also holds for the rank-one convex envelope of $W_{\text {cell }}^{\eta}$. To check this fact, it is enough to notice that by the test function in (2.8) we can obtain a suitable test function for the convex envelope of $t \mapsto W_{\text {cell }}\left(\Lambda+t \mathbf{e}_{1} \otimes \mathbf{e}_{2}\right)$ at $t=0$. In other words, a lamination in one single direction gives the upper bound on the rank-one convex envelope.

It is also worth noting that Proposition 1 is not peculiar to dimension two, as shown in the Appendix, although Remark 3 does not hold in dimension three.

2.4. Counterexample by comparison of the zero levelsets. Let us consider the following matrices of $\mathbb{M}^{2}$

$\mathbb{O}:=\operatorname{diag}(0,0), \mathbb{I}:=\operatorname{diag}(1,1), A:=\operatorname{diag}(-1,1), B:=\operatorname{diag}(0,1)$, and $C:=\operatorname{diag}(0,1 / 2)$,

and two quasiconvex functions $W_{1}, W_{2} \in \mathcal{W}(a, p)$ such that

$$
W_{1}^{-1}(0)=\{\mathbb{O}, A\} \text { and } W_{2}^{-1}(0)=\{\mathbb{O}, \mathbb{I}\} .
$$

We define $W: \mathbb{R}^{2} \times \mathbb{M}^{2} \rightarrow[0, \infty)$ by

$$
W(x, \Lambda):=\chi(x) W_{1}(\Lambda)+(1-\chi(x)) W_{2}(\Lambda),
$$

where $\chi$ is given by $\chi:=\chi_{(0,1 / 2) \times(0,1)}$ in $Q$ and extended by periodicity to the whole $\mathbb{R}^{2}$.

Then, Jean-François Babadjian and the first author proved in [3, Example 6.1] that $W_{\text {cell }}(\mathbb{I})=W_{\text {cell }}(B)=W_{\text {hom }}(\mathbb{I})=W_{\text {hom }}(B)=0$ and $W_{\text {cell }}(C)>0$. Since $C \in\{\mathbb{O}, B\}^{r c}$ and $W_{\text {hom }}$ is rank-one convex, this implies that $W_{\text {hom }}(C)=0<W_{\text {cell }}(C)$. However, one also has $Q W_{\text {cell }}(C)=0$.

\section{Counterexamples from composite materials}

In this section, we propose two examples for which $Q W_{\text {cell }}(\Lambda)>W_{\text {hom }}(\Lambda)$ for some $\Lambda \in \mathbb{M}^{2}$. The first one relies on the rigidity of periodic discrete gradients, whereas the second example uses the rigidity of the incompatible two-well problem together with the periodicity constraint. Both examples are based on the same geometry (see Figures 2 and 4). 
3.1. Discrete example. Let us first describe the geometry of the model.

Geometry. The geometry is a $Q$-periodic triangulation $\mathcal{T}$ of $\mathbb{R}^{2}$. The periodic pattern is sketched on Figure 2. We will make use of the following notation: For all $n \in \mathbb{N}$, let $\mathcal{T}_{n}=Q_{n} \cap \mathcal{T}$ while for all $m \in \mathbb{Z}^{2}$ and $\tau \in\{1, \ldots, 8\}, T_{\tau}^{m}$ denotes the $\tau^{\text {th }}$ triangle of $\mathcal{T} \cap Q^{m}$, according to the numerotation of Figure 2. Moreover, for $i \in\{1,2,3\}$, we denote by $x_{\tau, i}^{m}$ the $i^{t h}$ vertex of the triangle $T_{\tau}^{m}$ and we set $\mathcal{N}_{\tau}^{m}:=\left\{x_{\tau, 1}^{m}, x_{\tau, 2}^{m}, x_{\tau, 3}^{m}\right\}$.

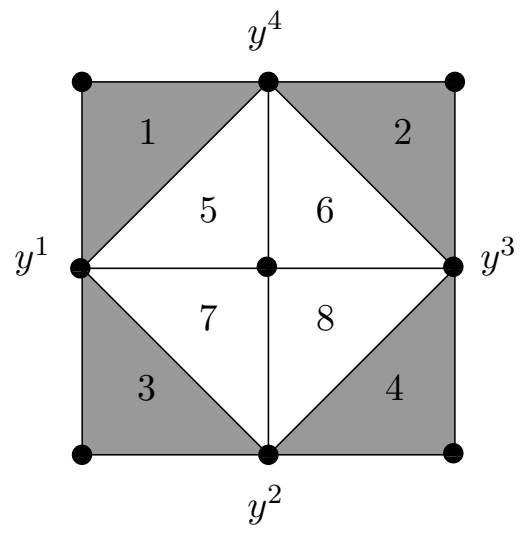

Figure 2. Geometry.

Energy. Let $U$ be a bounded open subset of $\mathbb{R}^{2}$. Given $u \in \mathcal{S}\left(U, \mathbb{R}^{2}\right), m \in \mathbb{Z}^{2}, \tau \in$ $\{1, \ldots, 8\}$, and $i \in\{1,2,3\}$, if $U \cap T_{\tau}^{m} \neq \varnothing$ and $x_{\tau, i}^{m} \in U$, we set

$$
\begin{aligned}
\nabla u_{\tau}^{m} & :=\nabla u_{\mid T_{\tau}^{m}}\left(\text { which is constant on } T_{\tau}^{m}\right) \\
u_{\tau, i}^{m} & :=u\left(x_{\tau, i}^{m}\right) .
\end{aligned}
$$

Let $f_{1}, f_{2}: \mathbb{R}^{2} \rightarrow[0,+\infty)$ be defined by

$$
\begin{aligned}
& f_{1}(z):=\left(z^{2}-1\right)^{2} \\
& f_{2}(z):=(z-1)^{2} .
\end{aligned}
$$

Accordingly to Definition 4 , for all $\eta>0$ we consider the energy

$$
F^{\eta}(u, U):=\sum_{m \in \mathbb{Z}^{2}: Q^{m} \subseteq U} F^{\eta, m}(u),
$$

where, for any $m \in \mathbb{Z}^{2}$ such that $Q^{m} \subseteq U$,

$$
\begin{aligned}
F^{\eta, m}(u) & :=\sum_{\tau=1}^{4}\left[\frac{1}{8} f_{2}\left(\operatorname{det} \nabla u_{\tau}^{m}\right)+\sum_{i, j \in \mathcal{N}_{\tau}^{m}, i<j} \frac{\eta}{2} f_{1}\left(\frac{\left|u_{\tau, i}^{m}-u_{\tau, j}^{m}\right|}{\left|x_{\tau, i}^{m}-x_{\tau, j}^{m}\right|}\right)\right] \\
& +\sum_{\tau=5}^{8}\left[\frac{\eta}{8} f_{2}\left(\operatorname{det} \nabla u_{\tau}^{m}\right)+\sum_{i, j \in \mathcal{N}_{\tau}^{m}, i<j} \frac{\eta}{2} f_{1}\left(\frac{\left|u_{\tau, i}^{m}-u_{\tau, j}^{m}\right|}{\left|x_{\tau, i}^{m}-x_{\tau, j}^{m}\right|}\right)\right] .
\end{aligned}
$$

The model satisfies the assumptions of Theorem 2 . We respectively denote by $W_{\text {cell }}^{\eta}$ and $W_{\text {hom }}^{\eta}$ the cell integrand and the homogenized integrand associated with $\left(\mathcal{T}, F^{\eta}\right)$.

The following results hold. 
Lemma 9. For all $\eta>0$ and for all $\Lambda \in \mathbb{M}^{2}$ invertible, $Q W_{\text {cell }}^{\eta}$ satisfies the following lower bound

$$
Q W_{\text {cell }}^{\eta}(\Lambda) \geq \frac{1}{2} f_{2}(\operatorname{det} \Lambda) .
$$

Lemma 10. For all $\lambda \geq 1$, there exists $c>0$ independent of $\eta$ such that

$$
W_{\text {hom }}^{\eta}(\lambda \mathbb{I}) \leq \eta c
$$

Theorem 4. For all $\lambda>1$, there exists $\eta>0$ such that

$$
W_{\text {hom }}^{\eta}(\lambda \mathbb{I})<Q W_{\text {cell }}^{\eta}(\lambda \mathbb{I}) \text {. }
$$

Theorem 4 is a direct consequence of Lemmas 9 and 10. Let us prove the two lemmas. Proof of Lemma 9. For all $\eta>0$, let consider the energy

$$
\widetilde{F}^{\eta}(u, U):=\sum_{m \in \mathbb{Z}^{2}: Q^{m} \subseteq U} \widetilde{F}^{\eta, m}(u),
$$

where, for any $m \in \mathbb{Z}^{2}$ such that $Q^{m} \subseteq U$,

$$
\widetilde{F}^{\eta, m}(u):=\sum_{\tau=1}^{4} \frac{1}{8} f_{2}\left(\operatorname{det} \nabla u_{\tau}\right)+\sum_{\tau=5}^{8} \frac{\eta}{8} f_{2}\left(\operatorname{det} \nabla u_{\tau}\right) .
$$

Let $\widetilde{W}_{\text {cell }}^{\eta}$ be the cell integrand associated with $\left(\mathcal{T}, \widetilde{F}^{\eta}\right)$. Since we have neglected the contributions of the terms involving $f_{1}$, we have $W_{\text {cell }}^{\eta} \geq \widetilde{W}_{\text {cell }}^{\eta}$.

For all $\eta>0$ and $\Lambda \in \mathbb{M}^{2}$, we claim that

$$
\widetilde{W}_{\text {cell }}^{\eta}(\Lambda)=\widetilde{F}^{\eta}\left(\varphi_{\Lambda}, Q\right) .
$$

Let $\psi$ be an admissible deformation of the form $\psi=\varphi_{\Lambda}+\phi, \phi \in \mathcal{S}_{\text {per }}\left(Q, \mathbb{R}^{2}\right)$. Due to the periodicity constraint on $Q$, an elementary geometric argument shows that

$$
\begin{aligned}
& \frac{1}{4}\left(\operatorname{det} \nabla \psi_{\mid T_{1}}+\operatorname{det} \nabla \psi_{\mid T_{2}}+\operatorname{det} \nabla \psi_{\mid T_{3}}+\operatorname{det} \nabla \psi_{\mid T_{4}}\right)=\operatorname{det} \Lambda, \\
& \frac{1}{4}\left(\operatorname{det} \nabla \psi_{\mid T_{5}}+\operatorname{det} \nabla \psi_{\mid T_{6}}+\operatorname{det} \nabla \psi_{\mid T_{7}}+\operatorname{det} \nabla \psi_{\mid T_{8}}\right)=\operatorname{det} \Lambda .
\end{aligned}
$$

To prove this assertion, up to multiplying $\nabla \psi$ by $\Lambda^{-1}$, it is enough to consider $\Lambda=\mathbb{I}$. In this case, referring to Figure 2, we define $\psi$ at $y^{1}:=(0,1 / 2), y^{2}:=(1 / 2,0), y^{3}:=$ $(1,1 / 2), y^{4}:=(1 / 2,1)$ by

$$
\begin{aligned}
& \psi\left(y^{1}\right)=\left(\alpha_{1}, 1 / 2+\beta_{1}\right) \\
& \psi\left(y^{3}\right)=\left(1+\alpha_{1}, 1 / 2+\beta_{1}\right), \\
& \psi\left(y^{2}\right)=\left(1 / 2+\alpha_{2}, \beta_{2}\right), \\
& \psi\left(y^{4}\right)=\left(1 / 2+\alpha_{2}, 1+\beta_{2}\right),
\end{aligned}
$$

where $\alpha_{1}, \alpha_{2}, \beta_{1}, \beta_{2} \in \mathbb{R}$. A straightforward calculation then shows that

$$
\begin{aligned}
& \operatorname{det} \nabla \psi_{\mid T_{1}}=4\left(\alpha_{1} \beta_{2}-\left(\beta_{1}-1 / 2\right)\left(\alpha_{2}+1 / 2\right)\right) \\
& \operatorname{det} \nabla \psi_{\mid T_{2}}=4\left(-\alpha_{1} \beta_{2}+\left(\beta_{1}-1 / 2\right)\left(\alpha_{2}-1 / 2\right)\right) \\
& \operatorname{det} \nabla \psi_{\mid T_{3}}=4\left(-\alpha_{1} \beta_{2}+\left(\beta_{1}+1 / 2\right)\left(\alpha_{2}+1 / 2\right)\right) \\
& \operatorname{det} \nabla \psi_{\mid T_{4}}=4\left(\alpha_{1} \beta_{2}-\left(\beta_{1}+1 / 2\right)\left(\alpha_{2}-1 / 2\right)\right) .
\end{aligned}
$$




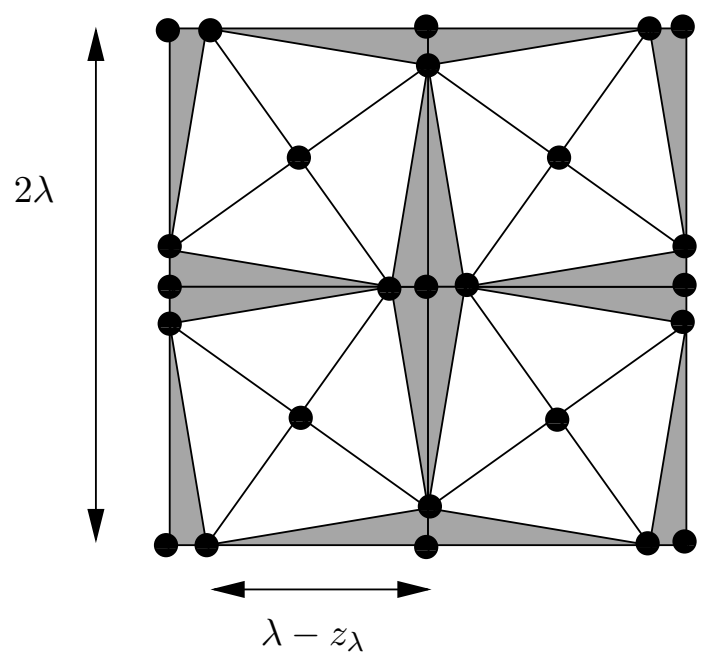

Figure 3. Deformation of $Q_{2}$ by the $Q_{2}$-periodic competitor $\psi$.

Thus, as expected,

$$
\frac{1}{4}\left(\operatorname{det} \nabla \psi_{\mid T_{1}}+\operatorname{det} \nabla \psi_{\mid T_{2}}+\operatorname{det} \nabla \psi_{\mid T_{3}}+\operatorname{det} \nabla \psi_{\mid T_{4}}\right)=1
$$

and the second equation of (3.1) follows now from the fact that $\int_{Q} \operatorname{det}(\Lambda+\nabla \phi)=\operatorname{det} \Lambda$ because \pm det is quasiconvex.

Hence, by Jensen's inequality ( $f_{2}$ is a convex function),

$$
\widetilde{W}_{\text {cell }}^{\eta}(\Lambda)=\frac{1}{2}(1+\eta) f_{2}(\operatorname{det} \Lambda) .
$$

Since $\widetilde{W}_{\text {cell }}^{\eta}$ is a polyconvex function (hence quasiconvex) not greater than $W_{\text {cell }}^{\eta}$ on $\mathbb{M}^{2}$, for all $\Lambda \in \mathbb{M}^{2}$ there holds

$$
Q W_{\text {cell }}^{\eta}(\Lambda) \geq \widetilde{W}_{\text {cell }}^{\eta}(\Lambda) \geq \frac{1}{2} f_{2}(\operatorname{det} \Lambda) .
$$

Proof of Lemma 10. Let $z_{\lambda}$ be a solution of $z_{\lambda}\left(\lambda-z_{\lambda}\right)=1 / 8$. We define a $Q_{2}$-periodic competitor $\psi$ as on Figure 3.

Since in triangles of the form $T_{i}^{m}, i \in\{1,2,3,4\}$, where the material is strong,

$$
\operatorname{det} \nabla \psi=1 \Longrightarrow f_{2}(\operatorname{det} \nabla \psi)=0,
$$

one has $F^{\eta}\left(\psi, Q_{2}\right)=\eta F^{1}\left(\psi, Q_{2}\right)$. Hence, since $\psi-\varphi_{\lambda \mathbb{I}} \in \mathcal{S}_{\text {per }}\left(Q_{2}, \mathbb{R}^{2}\right)$, we have

$$
W_{\text {hom }}^{\eta}(\lambda \mathbb{I}) \leq F^{\eta}\left(\psi, Q_{2}\right) \leq \eta F^{1}\left(\psi, Q_{2}\right) .
$$

3.2. An example from solid-solid phase transformations. To build the following counterexample, we introduce energy densities on $Q$ such that a phenomenon similar to the one on Figure 2 may occur at the continuous level. The rigidity now relies on the set of matrices we introduce hereafter. 


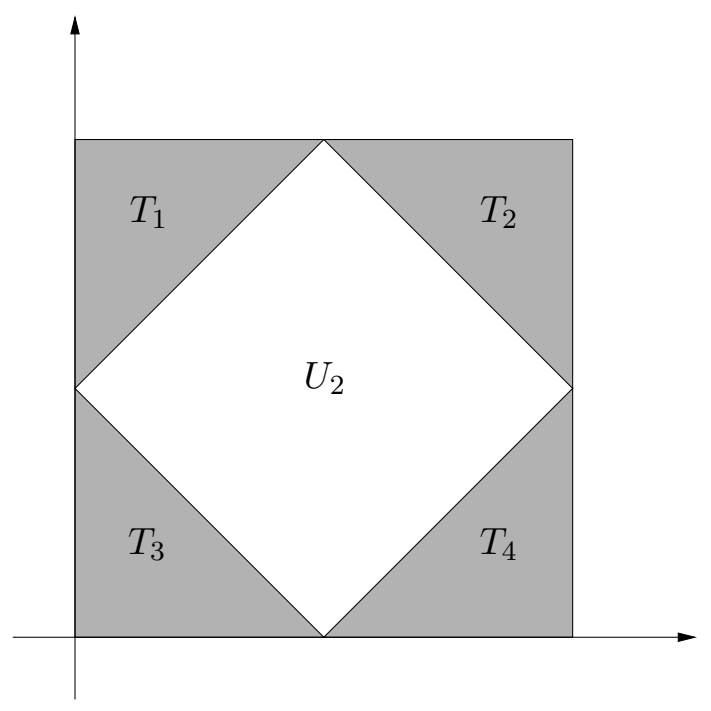

Figure 4. Geometry.

- Matrices in $\mathbb{M}^{2}$

$$
\begin{aligned}
& A_{1}:=\operatorname{diag}(1,1), A_{2}:=\operatorname{diag}(4,3), B_{1}:=\operatorname{diag}(1,3), B_{2}:=\operatorname{diag}(4,1), \\
& C:=\frac{1}{2} \operatorname{diag}(5,4), R:=\frac{1}{\sqrt{2}}\left(\begin{array}{cc}
1 & 1 \\
-1 & 1
\end{array}\right) .
\end{aligned}
$$

- Compact sets in $\mathbb{M}^{2}$

$$
\begin{array}{ll}
K_{1}:=S O_{2} A_{1} \cup S O_{2} A_{2}, & K_{2}:=S O_{2} B_{1} \cup S O_{2} B_{2}, \\
H_{1}:=K_{1} R, & H_{2}:=\left(K_{2} R\right)^{p c} .
\end{array}
$$

- Geometry (see Figure 4)

$$
\begin{array}{ll}
T_{1}:=\left\{x \in Q: x_{2} \geq x_{1}+1 / 2\right\}, & T_{2}:=\left\{x \in Q: x_{2} \geq-x_{1}+3 / 2\right\}, \\
T_{3}:=\left\{x \in Q: x_{2} \leq-x_{1}+1 / 2\right\}, & T_{4}:=\left\{x \in Q: x_{2} \leq x_{1}-1 / 2\right\}, \\
U_{1}:=\bigcup_{i=1}^{4} T_{i}, & U_{2}:=Q \backslash U_{1} .
\end{array}
$$

The counterexample is as follows.

Theorem 5. Let $W_{1}, W_{2} \in \mathcal{W}(a, p)$ be two quasiconvex functions (to be built later) such that

$$
W_{1}^{-1}(0)=H_{1} \quad \text { and } \quad W_{2}^{-1}(0)=H_{2} .
$$

Consider the energy density $W: \mathbb{R}^{2} \times \mathbb{M}^{2} \rightarrow[0,+\infty)$ defined by

$$
W(x, \Lambda):=\chi(x) W_{1}(\Lambda)+(1-\chi(x)) W_{2}(\Lambda),
$$

where $\chi$ is given by $\chi:=\chi_{U_{1}}$ in $Q$ and extended by periodicity to the whole $\mathbb{R}^{2}$. The following properties hold:

1) the cell integrand $W_{\text {cell }}$ related to $W$ is bounded from below by a constant $c>0$;

2) $C R$ belongs to the zero levelset of the homogenized integrand $W_{\text {hom }}$ related to $W$. Therefore $Q W_{\text {cell }}(C R) \geq c>W_{\text {hom }}(C R)$.

We will make use of the following facts in the proof. 
i) The compact set $K_{1}$ is polyconvex and rigid, i.e., if $U \subseteq \mathbb{R}^{2}$ is an open connected set and $\psi: U \rightarrow \mathbb{R}^{2}$ is a Lipschitz function such that

$$
\nabla \psi(x) \in K_{1} \quad \text { for a.e. } x \in U,
$$

then $\psi$ is affine. We refer to [15, Theorem 2] and [13, Theorem 4.11] for the proofs. Since $R$ is a rotation, the same properties hold for $H_{1}$.

ii) $H_{1} \cap H_{2}=\varnothing$, because by Lemma 4

$$
H_{2} \subseteq\left\{\Lambda \in \mathbb{M}^{2}: \operatorname{det} \Lambda \in[3,4]\right\} .
$$

iii) $A_{1}$ is rank-one connected to $B_{1}$ and $B_{2}$, and $A_{2}$ to $B_{1}$ and $B_{2}$ also. More precisely, denoted by $\left\{\mathbf{e}_{1}, \mathbf{e}_{2}\right\}$ the canonical basis in $\mathbb{R}^{2}$,

$$
\begin{aligned}
& A_{1}-B_{1}=-2 \mathbf{e}_{2} \otimes \mathbf{e}_{2} \\
& A_{1}-B_{2}=-3 \mathbf{e}_{1} \otimes \mathbf{e}_{1} \\
& A_{2}-B_{1}=3 \mathbf{e}_{1} \otimes \mathbf{e}_{1} \\
& A_{2}-B_{2}=2 \mathbf{e}_{2} \otimes \mathbf{e}_{2} .
\end{aligned}
$$

Proof of Theorem 5.

Property 1). Since $W_{\text {cell }}$ grows superlineary at infinity and is continuous by Lemma 6 , it is enough to prove that $W_{\text {cell }}(\Lambda) \neq 0$ for any $\Lambda \in \mathbb{M}^{2}$. We proceed by contradiction and assume there exists $\Lambda \in \mathbb{M}^{2}$ such that $W_{\text {cell }}(\Lambda)=0$. By Lemma 8 , there exists a $Q$-periodic Lipschitz function $\phi: \mathbb{R}^{2} \rightarrow \mathbb{R}^{2}$ such that

$$
\Lambda+\nabla \phi(x) \in\left\{\begin{array}{ll}
H_{1} & \text { for a.e. } x \in U_{1} \\
H_{2} & \text { for a.e. } x \in U_{2}
\end{array} .\right.
$$

Due to the rigidity, we infer that there exists $D_{i} \in H_{1}$ such that $\Lambda+\nabla \phi(x)=D_{i}$ for a.e. $x \in T_{i}$. Again by the rigidity, the periodicity condition implies that there exists $D \in H_{1}$ such that $D_{i}=D$ for all $i \in\{1,2,3,4\}$.

By observing that $\psi(x):=(\Lambda-D) \cdot x+\phi(x)$ belongs to $W_{0}^{1, \infty}\left(U_{2}\right)$, from the definition of quasiconvexity we get

$$
W_{2}(D) \leq f_{U_{2}} W_{2}(D+\nabla \psi(x)) d x=f_{U_{2}} W_{2}(\Lambda+\nabla \phi(x)) d x=0
$$

and so $D \in H_{2}$, which contradicts $H_{1} \cap H_{2}=\varnothing$.

Property 2). It is sufficient to find $\phi \in W_{\text {per }}^{1, p}\left(Q_{2}, \mathbb{R}^{2}\right)$ such that

$$
\int_{Q_{2}} W(x, C R+\nabla \phi(x)) d x=0 .
$$

This can be accomplished by using the following function $\psi:(-1 / \sqrt{2}, 1 / \sqrt{2})^{2} \rightarrow \mathbb{R}^{2}$,

$$
\begin{aligned}
& \psi^{(1)}(x):=\left\{\begin{array}{ll}
x_{1} & \text { if } x \in(-1 / \sqrt{2},-\sqrt{2} / 4) \times(-1 / \sqrt{2}, 1 / \sqrt{2}) \\
4 x_{1}-3 \sqrt{2} / 4 & \text { if } x \in[-\sqrt{2} / 4, \sqrt{2} / 4] \times(-1 / \sqrt{2}, 1 / \sqrt{2}) \\
x_{1}+3 \sqrt{2} / 2 & \text { if } x \in(\sqrt{2} / 4,1 / \sqrt{2}) \times(-1 / \sqrt{2}, 1 / \sqrt{2})
\end{array} ;\right. \\
& \psi^{(2)}(x):=\left\{\begin{array}{ll}
x_{2} & \text { if } x \in(-1 / \sqrt{2}, 1 / \sqrt{2}) \times(-1 / \sqrt{2},-\sqrt{2} / 4) \\
3 x_{2}-\sqrt{2} / 2 & \text { if } x \in(-1 / \sqrt{2}, 1 / \sqrt{2}) \times[-\sqrt{2} / 4, \sqrt{2} / 4] \\
x_{2}+\sqrt{2} & \text { if } x \in(-1 / \sqrt{2}, 1 / \sqrt{2}) \times(\sqrt{2} / 4,1 / \sqrt{2})
\end{array} .\right.
\end{aligned}
$$


Let $\varphi$ be the $(-1 / \sqrt{2}, 1 / \sqrt{2})^{2}$-periodic extension of $\varphi: x \mapsto \psi(x)-C \cdot x$. Then $\phi: x \mapsto$ $\varphi(R \cdot x)$ does the job. Actually, as illustrated on Figure 5, $C R+\nabla \phi(x+m) \in H_{1}$ for $(x, m) \in U_{1} \times \mathbb{Z}^{2}$ and $C R+\nabla \phi(x+m) \in H_{2}$ for $(x, m) \in U_{2} \times \mathbb{Z}^{2}$.

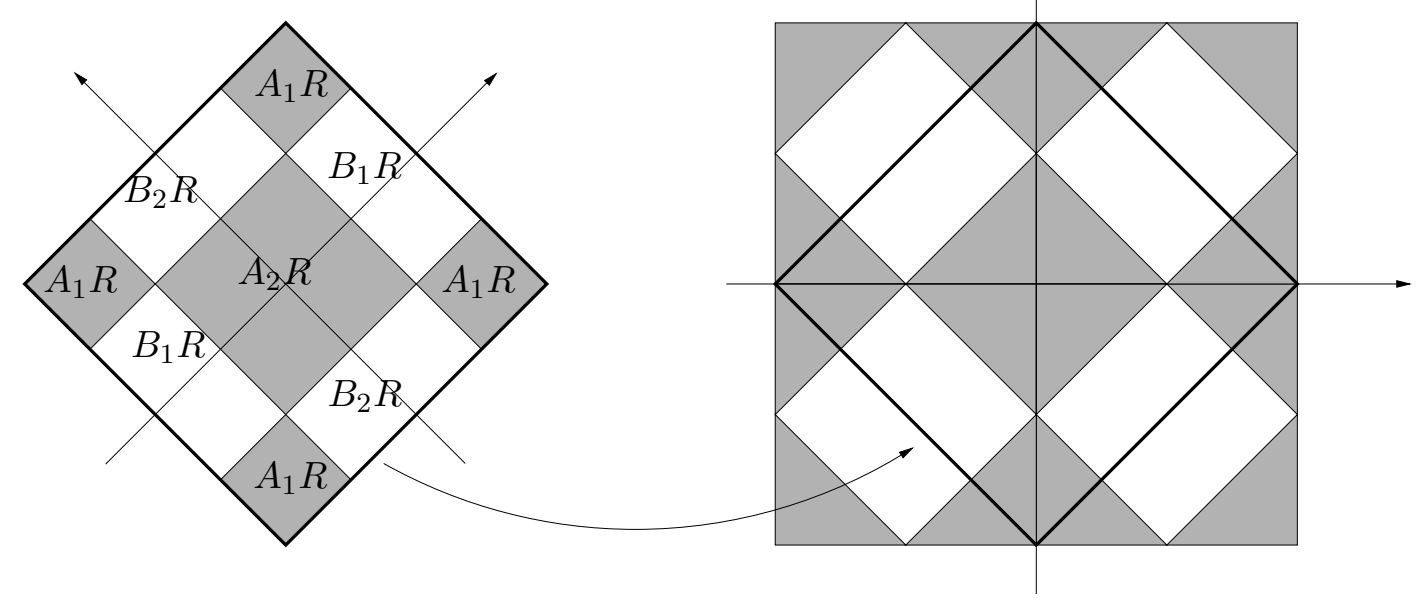

Figure 5. The values of $C R+\nabla \phi$ in $R^{-1}(-1 / \sqrt{2}, 1 / \sqrt{2})^{2}$. On the left the axis are oriented in the directions $R^{-1} \mathbf{e}_{1}$ and $R^{-1} \mathbf{e}_{2}$.

To complete the counterexample we need to build two quasiconvex functions $W_{1}, W_{2}$ satisfying (3.2).

Lemma 11. Let $H$ be a compact, polyconvex and frame-invariant subset of $\mathbb{M}^{2}$. Given $p \in(1,+\infty)$, for a suitable $a>0$ there exists a quasiconvex function $W \in \mathcal{W}(a, p)$ such that

$$
W^{-1}(0)=H .
$$

If $p \geq 2$, then $W$ can be chosen polyconvex.

Proof. Let $V(\Lambda):=\operatorname{dist}(\Lambda, H)^{p}$ and set $W(\Lambda):=Q V(\Lambda)$. By Lemma $5, W^{-1}(0)=H$. Since the Frobenius norm is frame-invariant, the same holds for $V$, and therefore for $W$ since for all $\Lambda \in \mathbb{M}^{2}$ and all $R \in S_{2}$,

$$
\begin{aligned}
W(R \Lambda) & =\inf \left\{\int_{Q} V(R \Lambda+\nabla \phi) d x: \phi \in W_{0}^{1, p}\left(Q, \mathbb{R}^{2}\right)\right\} \\
& =\inf \left\{\int_{Q} V\left(R \Lambda+R \nabla R^{-1} \phi\right) d x: \phi \in W_{0}^{1, p}\left(Q, \mathbb{R}^{2}\right)\right\} \\
& =\inf \left\{\int_{Q} V(R(\Lambda+\nabla \varphi)) d x: \varphi \in W_{0}^{1, p}\left(Q, \mathbb{R}^{2}\right)\right\} \\
& =\inf \left\{\int_{Q} V(\Lambda+\nabla \varphi) d x: \varphi \in W_{0}^{1, p}\left(Q, \mathbb{R}^{2}\right)\right\}=W(\Lambda) .
\end{aligned}
$$


A different construction allows us to consider a polyconvex energy density in the case $p \in[2,+\infty)$. We define the functions

$$
V_{1}(\Lambda):=\operatorname{dist}\left(\Lambda, H^{c o}\right)^{p} \quad \text { and } \quad V_{2}(\Lambda):=\operatorname{dist}(\mathbf{M}(\Lambda), L)^{\frac{p}{2}},
$$

where $L:=\{\mathbf{M}(\Lambda): \Lambda \in H\}^{c o}$. Both are polyconvex and with $p$-growth, moreover $V_{1}$ is $p$-coercive and, by Lemma 4 , the zero levelset of $V_{2}$ is $H$. The function $W:=\max \left\{V_{1}, V_{2}\right\}$ does the job. In addition, it is easy to verify that also in this case $W$ is frame-invariant.

Remark 4. The previous lemma is optimal, because a polyconvex function with subquadratic growth is convex (see [8, Corollary 5.9]).

3.3. Comparison of boths examples. In the discrete example, the zero levelset of the energy density of the strong phase is $\mathcal{J}=\left\{\Lambda \in \mathbb{M}^{2}\right.$, $\left.\operatorname{det} \Lambda=1\right\}$, the space of isochoric deformations, which is not rigid. The rigidity comes from the structure of $Q$-periodic discrete gradients on $\mathcal{T}_{1}$.

In the continuous example, we replace $\mathcal{S}_{\text {per }}^{1}\left(Q, \mathbb{R}^{2}\right)$ by $W_{\text {per }}^{1, p}\left(Q, \mathbb{R}^{2}\right)$, hence adding much more flexibility to the periodic gradients. In order to keep the required rigidity, we then replace $\mathcal{J}$ by $H_{1}$ in the strong phase.

The rigidity of the discrete example lies in (3.1), whereas the rigidity of the continuous example lies in (3.3).

Compared to Stefan Müller's example, the repartition of the strong phase in $Q$ allows to take full advantage of the constraint of periodicity in both examples of this section. On the contrary, in Section 2.3, the periodicity constraint is lost in the $x_{1}$-direction, as shown by Proposition 1.

\section{Counterexample on Perforated domains}

\section{Geometry and energy}

Let us begin by describing the geometry of the subset $P$, sketched on Figure 6 .

Definition 11. The set $P$ is the complement in $\mathbb{R}^{2}$ of the set $\bigcup_{m \in \mathbb{Z}^{2}} O+m$, where

$$
O:=\left\{x \in Q: x_{1} \in[1 / 8,7 / 8] \text { and } 3 x_{1} \leq 4 x_{2} \leq 3 x_{1}+1\right\} .
$$

Let us introduce some sets in the space $\mathbb{M}^{2}$, that we will use to describe the energy density.

Definition 12. Let $l_{1}:=3 / 4, l_{2}:=9 / 16$ and $l_{3}:=1 / 4$. We consider the following sets (see Figure 7):

- $K_{1}:=\{\operatorname{diag}(s, 0): s \in(0,1]\}$ and $K_{2}:=\{\operatorname{diag}(0, t): t \in(0,1]\}$

- $K:=K_{1} \cup K_{2} \cup\{\operatorname{diag}(0,0)\}$;

- $H:=\{\operatorname{diag}(s, t): s, t \in[0,1]$ and $t \leq 1-s\}$;

- $L:=\left\{\operatorname{diag}(s, t): 0<s \leq l_{1} l_{3} / l_{2}\right.$ and $\left.0<t \leq l_{3}-l_{2} s / l_{1}\right\}$;

- $M:=\left\{\operatorname{diag}(s, t): 0<s \leq l_{1} / 2\right.$ and $\left.0<t \leq l_{3}\right\}$.

The counterexample is as follows.

Theorem 6. Let $p \in[2,+\infty)$ and let $W: \mathbb{M}^{2} \rightarrow[0,+\infty)$ be given by

$$
W(\Lambda):=\operatorname{dist}(\Lambda, H)^{p}+|\operatorname{det}(\Lambda)|^{\frac{p}{2}},
$$




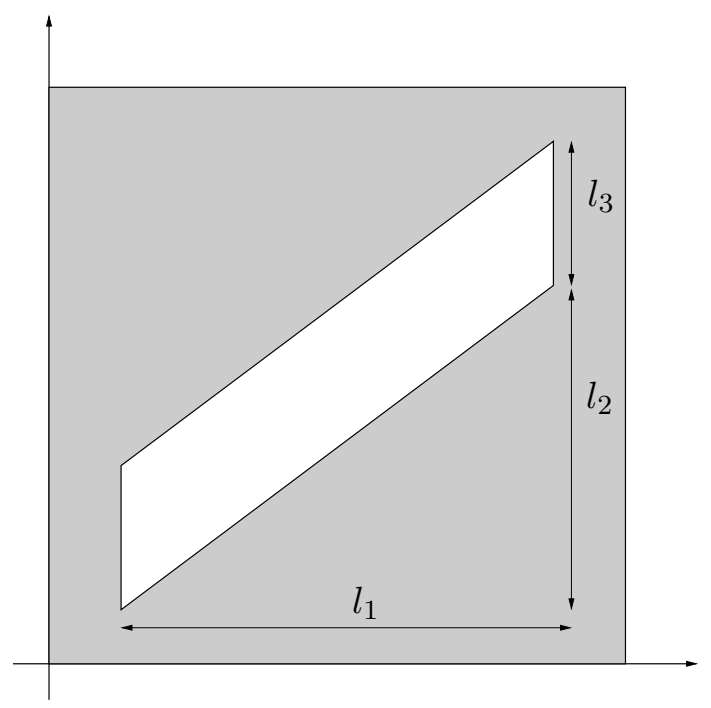

Figure 6. In grey the set $Q \cap P$.
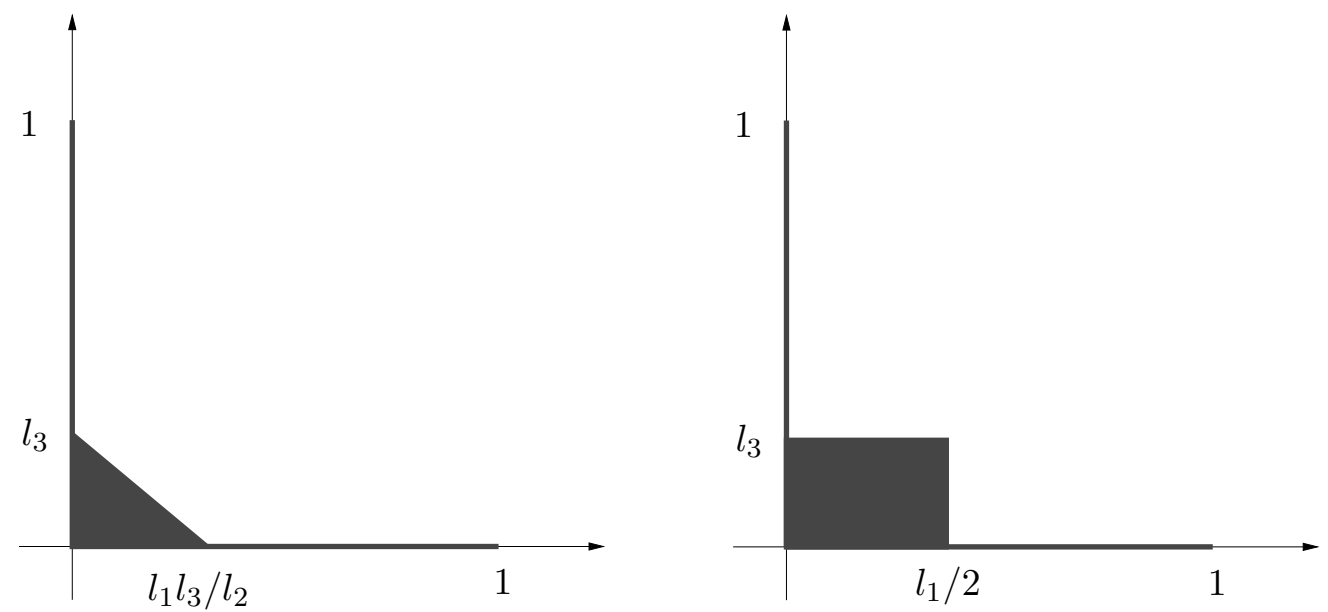

Figure 7. A representation of $K \cup L$ and $K \cup M$ in $\mathbb{R}^{2}$, identified with the set of the diagonal matrices.

where $H$ is as in Definition 12. Then, $W$ is polyconvex and belongs to $\mathcal{W}(a, p)$ for a suitable $a>0$. Moreover, with the notation of Definitions 11 and 12, there holds

1 ) the zero levelset of the cell integrand $W_{\text {cell }}$ related to $(W, P)$ coincides with $K \cup L$;

2 ) the zero levelset of the homogenized integrand $W_{\text {hom }}$ related to $(W, P)$ contains $K \cup M$;

3) $K \cup L$ is quasiconvex.

Therefore, for all $\Lambda \in(K \cup M) \backslash(K \cup L) \neq \varnothing, Q W_{\text {cell }}(\Lambda)>W_{\text {hom }}(\Lambda)=0$.

Proof. The set $H$ being convex, $\Lambda \mapsto \operatorname{dist}(\Lambda, H)^{p}$ is a convex function. Since $\Lambda \mapsto|\operatorname{det} \Lambda|^{\frac{p}{2}}$ is polyconvex, $W$ is polyconvex. It is easy to verify that $W$ satisfies $(2.1)$ for a suitable 
$a>0$. The strict inequality $Q W_{\text {cell }}(\Lambda)>W_{\text {hom }}(\Lambda)$ is a direct consequence of 1$\left.)-3\right)$ using Lemma 5. Let us split the proof of 1)-3) into three steps.

Step 1. Since $W^{-1}(0)=K$, by using $\phi \equiv 0$ as a test function in (2.2), we obtain $K \subseteq W_{\text {cell }}^{-1}(0)$. Let us check that $L \subseteq W_{\text {cell }}^{-1}(0)$. Given $s \in\left(0, l_{1} l_{3} / l_{2}\right)$ and $t \in\left(0, l_{3}-l_{2} s / l_{1}\right)$, we define $\psi: Q \rightarrow \mathbb{R}^{2}$ by

$$
\begin{aligned}
& \psi^{(1)}(x):= \begin{cases}0 & \text { if } x \in(0,1 / 2) \times(0,1) \\
x_{1}-1 / 2 & \text { if } x \in[1 / 2,1 / 2+s] \times(0,1) \\
s & \text { if } x \in(1 / 2+s, 1) \times(0,1)\end{cases} \\
& \psi^{(2)}(x):= \begin{cases}0 & \text { if } x \in(0,1) \times(0,5 / 8-t) \\
x_{2}-5 / 8+t & \text { if } x \in(0,1) \times[5 / 8-t, 5 / 8] . \\
t & \text { if } x \in(0,1) \times(5 / 8,1)\end{cases}
\end{aligned}
$$

We have that $\phi(x):=\psi(x)-\operatorname{diag}(s, t) \cdot x$ is $Q$-periodic and that $\nabla \psi(x) \in K$ if $x \in P$. More precisely, $\nabla \psi(x) \notin K$ only if $x$ belongs to $[1 / 2,1 / 2+s] \times[5 / 8-t, 5 / 8] \subseteq O$. There, $\nabla \psi \equiv \operatorname{diag}(1,1)$ (see Figures 8 and 9 ).

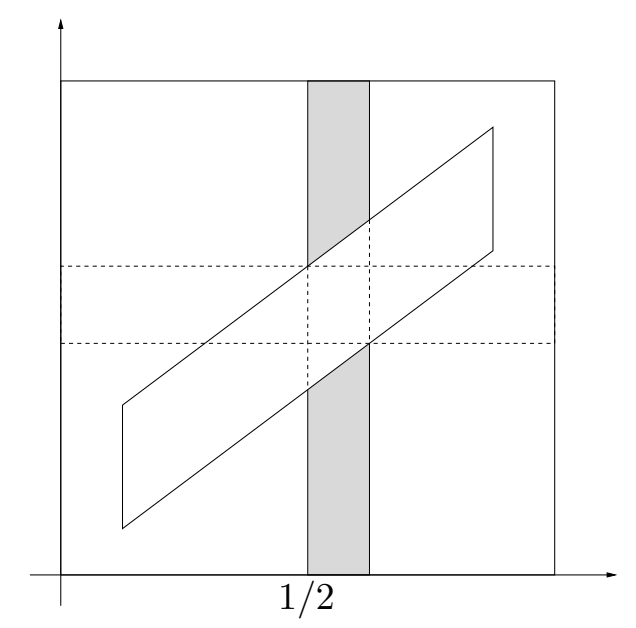

Figure 8. The first component of $\psi$ is flat on $Q \cap P$ with the exception of the grey zone, where the gradient is equal to $(1,0)$.

It remains to proceed with the delicate part of the argument: the opposite inclusion $W_{\text {cell }}^{-1}(0) \subseteq K \cup L$. Let $C=\left(c_{i j}\right) \in W_{\text {cell }}^{-1}(0)$. By Lemma 7, there exists a Lipschitz function $\psi: Q \rightarrow \mathbb{R}^{2}$ such that $\nabla \psi(x) \in K$ for $\mathcal{L}^{2}$ a.e. $x \in Q \cap P$ and $\phi(x):=\psi(x)-C \cdot x$ is $Q$-periodic. We will show that $\psi$ is substantially a laminate as in (4.1). Let us point out that if $\Lambda \in K$, then either $\Lambda_{11}=0$ or $\Lambda_{22}=0$.

We use the following notation:

$$
\begin{aligned}
& L^{s}:=\{r \in(0,1):(s, r) \in(\{s\} \times(0,1)) \cap P\} ; \\
& L_{s}:=\{r \in(0,1):(r, s) \in((0,1) \times\{s\}) \cap P\} .
\end{aligned}
$$

Notice that $L^{s}=(0,1)$ if $s \in(0,1 / 8) \cup(7 / 8,1)$ and that $L^{s}$ has two connected components if $s \in[1 / 8,7 / 8]$. Similarly, $L_{s}=(0,1)$ if $s \in(0,3 / 32) \cup(29 / 32,1)$ and it has two connected components if $s \in[3 / 32,29 / 32]$. 


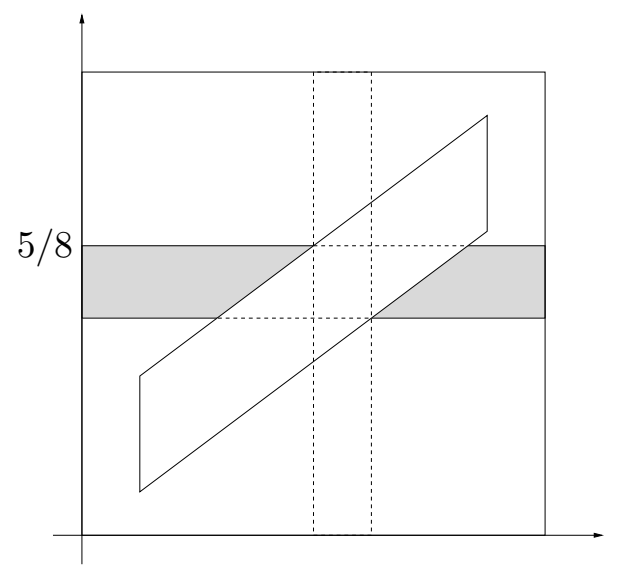

Figure 9. The second component of $\psi$ is flat on $Q \cap P$ with the exception of the grey zone, where the gradient is equal to $(0,1)$.

Since $\partial_{2} \psi^{(1)}(x)=0$ for $\mathcal{L}^{2}$ a.e. $x \in Q \cap P, \psi^{(1)}(s, \cdot)$ is constant along any connected component of $L^{s}$ for all $s \in(0,1)$. In particular for $s \in(0,1 / 8) \cup(7 / 8,1), \psi^{(1)}(s, \cdot)$ is constant and therefore the $(0,1)$-periodicity of $\phi(s, \cdot)$ imposes that $c_{12}=0$. For $s \in$ $[1 / 8,7 / 8], \psi^{(1)}(s, \cdot)$ is constant on each of the two connected components of $L^{s}$. Hence, by periodicity, $\psi^{(1)}(s, \cdot)$ is constant on the whole $L^{s}$.

From the fact that $\psi^{(1)}(s, \cdot)$ is constant along $L^{s}$ for any $s \in(0,1)$, we can deduce that if $\bar{x} \in Q \cap P$ is a differentiability point for $\psi^{(1)}$, then $\psi^{(1)}$ is differentiable in all $\left\{\bar{x}_{1}\right\} \times L^{\bar{x}_{1}}$ and

$$
\nabla \psi^{(1)}(x)=\nabla \psi^{(1)}(\bar{x}) \quad \forall x \in\left\{\bar{x}_{1}\right\} \times L^{\bar{x}_{1}} .
$$

Similarly, one can show that $c_{21}=0$ and that if $\bar{x} \in Q \cap P$ is a differentiability point for $\psi^{(2)}$, then $\psi^{(2)}$ is differentiable in all $L_{\bar{x}_{2}} \times\left\{\bar{x}_{2}\right\}$ and

$$
\nabla \psi^{(2)}(x)=\nabla \psi^{(2)}(\bar{x}) \quad \forall x \in L^{\bar{x}_{2}} \times\left\{\bar{x}_{2}\right\} .
$$

Let $X_{1}, X_{2}$ be two $\mathcal{L}^{1}$-negligible subsets of the interval $(0,1)$ such that $\psi$ is differentiable in $\widetilde{P}:=(Q \cap P) \backslash\left(X_{1} \times X_{2}\right)$ and $\nabla \psi(x) \in K$ for all $x \in \widetilde{P}$. Let us show that, if for some $\bar{x} \in \widetilde{P}$ there holds $\nabla \psi(\bar{x}) \in K_{1}$, then

$$
\nabla \psi(x) \in K_{1} \cup\{\operatorname{diag}(0,0)\} \quad \forall x \in \widetilde{P} \cap\left((0,1) \times L^{\bar{x}_{1}}\right) .
$$

In fact, since $\nabla \psi^{(1)}(\bar{x}) \neq(0,0)$, we have $\nabla \psi^{(1)} \neq(0,0)$ in $\left\{\bar{x}_{1}\right\} \times L^{\bar{x}_{1}}$ due to $(4.2)$ and therefore $\nabla \psi \in K_{1}$ in $\left\{\bar{x}_{1}\right\} \times\left(L^{\bar{x}_{1}} \backslash X_{2}\right)$. As now $\nabla \psi^{(2)} \equiv(0,0)$ in $\left\{\bar{x}_{1}\right\} \times\left(L^{\bar{x}_{1}} \backslash X_{2}\right)$, (4.3) implies that $\nabla \psi^{(2)} \equiv(0,0)$ in $\widetilde{P} \cap\left((0,1) \times L^{\bar{x}_{1}}\right)$.

We are in position to conclude the first step. Given $\bar{s} \in(0,1 / 8) \backslash X_{1}$ and $\bar{t} \in(0,3 / 32) \backslash$ $X_{2}$, we define the following two sets.

$$
\begin{aligned}
& S:=\left\{s \in(0,1) \backslash X_{1}: \partial_{1} \psi^{(1)}(s, \bar{t})>0\right\} ; \\
& T:=\left\{t \in(0,1) \backslash X_{2}: \partial_{2} \psi^{(2)}(\bar{s}, t)>0\right\} .
\end{aligned}
$$

Since $\nabla \psi \in K, \partial_{1} \psi^{(1)}(s, \bar{t}) \leq 1$ for all $s \in S$, and we infer from

$$
c_{11}=\int_{0}^{1} c_{11}+\partial_{1} \phi^{(1)}(s, \bar{t}) d s=\int_{0}^{1} \partial_{1} \psi^{(1)}(s, \bar{t}) d s=\int_{S} \partial_{1} \psi^{(1)}(s, \bar{t}) d s,
$$


that $0 \leq c_{11} \leq \mathcal{L}^{1}(S)$. Similarly, $0 \leq c_{22} \leq \mathcal{L}^{1}(T)$. In particular this shows that

$$
c_{11}, c_{22} \in[0,1] \text {. }
$$

If $c_{11}>0$, for any $\varepsilon>0$ there exist $s_{1}, s_{2} \in S$ such that $s_{2}-s_{1} \geq c_{11}-\varepsilon$. Recalling that if $\nabla \psi(x) \in K$ and $\partial_{1} \psi^{(1)}(x)>0$ then $\nabla \psi(x) \in K_{1}$, from (4.4), we obtain

$$
\nabla \psi(x) \in K_{1} \cup\{\operatorname{diag}(0,0)\} \quad \forall x \in \widetilde{P} \cap\left((0,1) \times\left(L^{s_{1}} \cup L^{s_{2}}\right)\right) .
$$

Since $T \subseteq(0,1) \backslash\left(L^{s_{1}} \cup L^{s_{2}}\right)$, we have the estimate

$$
c_{22} \leq \mathcal{L}^{1}(T) \leq \max \left\{0, \frac{l_{2}}{l_{1}}\left(s_{1}-s_{2}\right)+l_{3}\right\} \leq \max \left\{0, \frac{l_{2}}{l_{1}}\left(-c_{11}+\varepsilon\right)+l_{3}\right\} .
$$

The arbitrariness of $\varepsilon>0$ completes the proof of the step.

Step 2. Since $W_{\text {hom }}$ is rank-one convex and $W_{\text {hom }}^{-1}(0) \supseteq K$, it is sufficient to prove that

$$
C:=\operatorname{diag}\left(l_{1} / 2, l_{3}\right) \in W_{\text {hom }}^{-1}(0) .
$$

Let us construct a Lipschitz function $\psi: Q_{2} \rightarrow \mathbb{R}^{2}$ such that $\nabla \psi \in K$ a.e. in $Q_{2} \cap P$ and $\phi(x):=\psi(x)-C \cdot x$ is $Q_{2}$-periodic. In this way we get

$$
W_{\mathrm{hom}}(C) \leq \frac{1}{4} \int_{Q_{2} \cap P} W(C+\nabla \phi(x)) d x=0 .
$$

Despite the complexity of the following description, the function $\psi$ is very simple.

Consider the following sets (see Figure 10):

- $U:=\left\{x \in[1 / 8,1] \times\left[3 / 32,3 / 32+l_{2}\right]: 4 x_{2}<3 x_{1}\right\}$;

- $U_{1}:=\left([0,1] \times\left[0,3 / 32+l_{2}\right]\right) \backslash(U \cup O)$;

- $U_{2}:=\left\{x \in[1 / 8,1] \times\left[3 / 32,3 / 32+l_{3}\right]: 4 x_{2}<3 x_{1}\right\}$;

- $U_{3}:=\left\{x \in[0,1]^{2}:(1,1)-x \in U_{2}\right\}$;

- $U_{4}:=\left\{x \in[0,1]^{2}:(1,1)-x \in U_{1}\right\}$;

- $U_{5}:=U \cup([0,1] \times[0,1 / 8))$;

- $U_{6}:=[0,1 / 8) \times\left[3 / 32,3 / 32+l_{3}\right)$;

- $U_{7}:=\left\{x \in[0,1]^{2}:(1,1)-x \in U_{6}\right\}$;

- $U_{8}:=\left\{x \in[0,1]^{2}:(1,1)-x \in U_{5}\right\}$.

To simplify the exposition, we introduce two auxiliary Lipschitz functions $\varphi_{1}, \varphi_{2}:[0,1]^{2} \backslash$ $O \rightarrow \mathbb{R}:$

$$
\varphi_{1}(x):=\left\{\begin{array}{lll}
0 & \text { if } x \in U_{1} \\
x_{2}-3 / 32 & \text { if } x \in U_{2} \\
x_{2}-l_{2}-3 / 32 & \text { if } x \in U_{3} \\
h_{3} & \text { if } x \in U_{4}
\end{array} ; \quad \varphi_{2}(x):=\left\{\begin{array}{ll}
0 & \text { if } x \in U_{5} \\
x_{2}-3 / 32 & \text { if } x \in U_{6} \\
x_{2}-l_{2}-3 / 32 & \text { if } x \in U_{7} \\
h_{3} & \text { if } x \in U_{8}
\end{array} .\right.\right.
$$

Both $\varphi_{1}$ and $\varphi_{2}$ can be extended to Lipschitz maps in all $[0,1]^{2}$. 

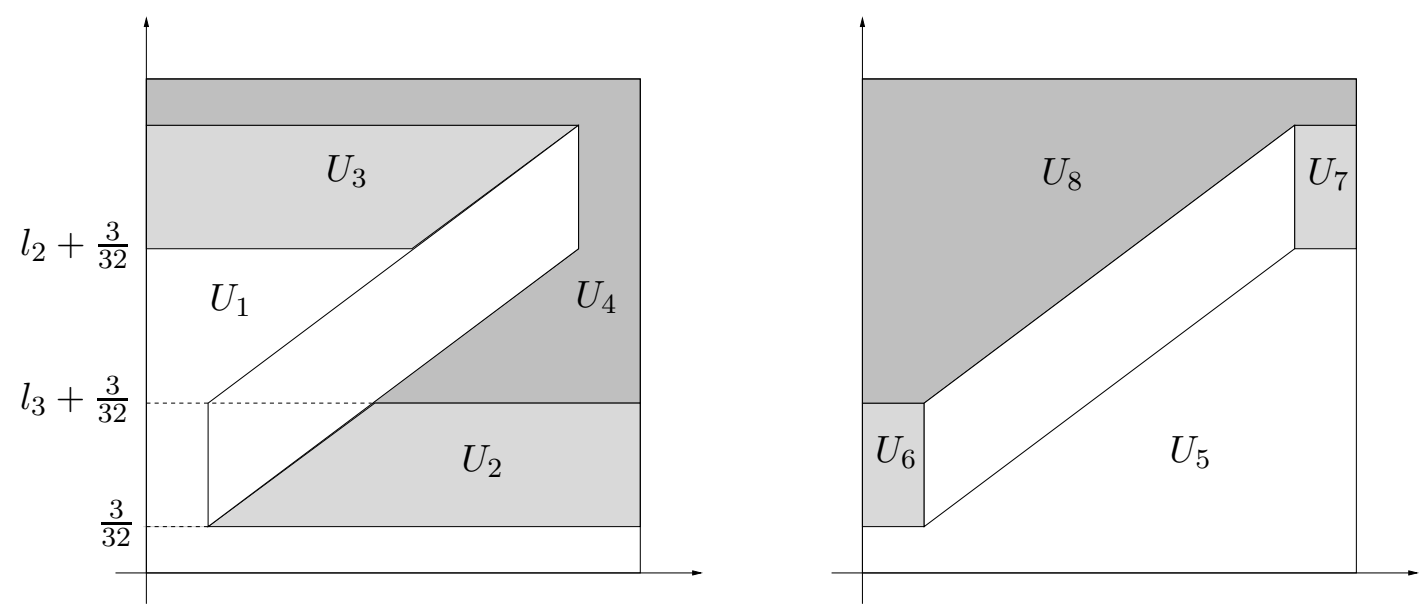

FiguRE 10

We are now in position to define the desired function $\psi: Q_{2} \rightarrow \mathbb{R}^{2}$ (see Figures 11, 12 and 13).

$$
\begin{aligned}
& \psi^{(1)}(x):= \begin{cases}0 & \text { if } x \in(0,9 / 8] \times(0,2) \\
x_{1}-9 / 8 & \text { if } x \in\left(9 / 8,9 / 8+l_{1}\right] \times(0,2) \\
l_{1} & \text { if } x \in\left(9 / 8+l_{1}, 2\right) \times(0,2)\end{cases} \\
& \psi^{(2)}(x):= \begin{cases}\varphi_{1}(x) & \text { if } x \in(0,1] \times(0,1] \\
\varphi_{2}(x-(1,0)) & \text { if } x \in(1,2) \times(0,1] \\
\varphi_{1}(x-(0,1))+l_{3} & \text { if } x \in(0,1] \times(1,2) \\
\varphi_{2}(x-(1,1))+l_{3} & \text { if } x \in(1,2) \times(1,2)\end{cases}
\end{aligned}
$$

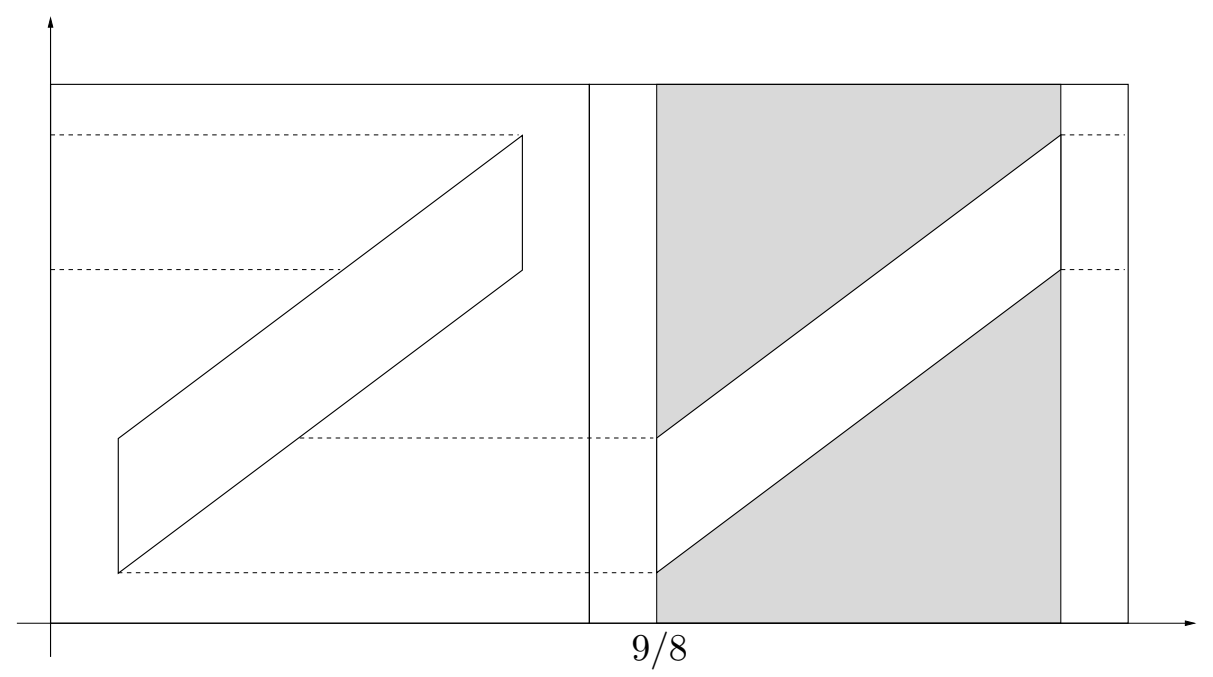

Figure 11. In $[(0,2) \times(0,1)] \cap P$ the first component of $\psi$ is flat with the exception of the grey zone, where the gradient is equal to $(1,0)$. 


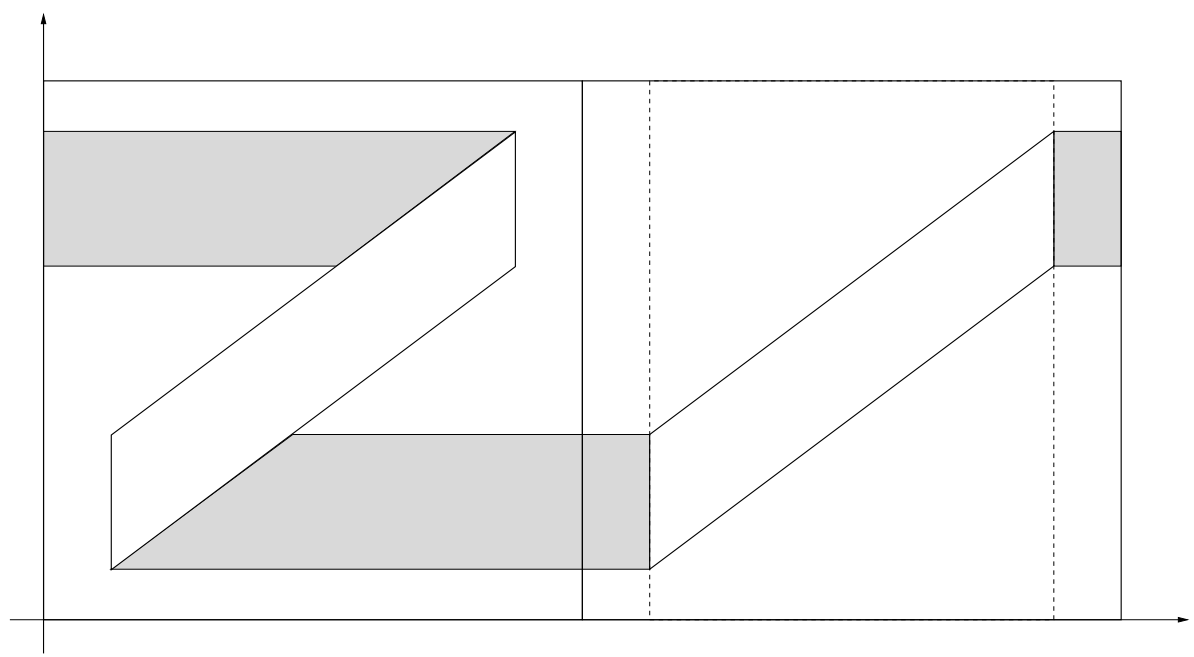

Figure 12 . In $[(0,2) \times(0,1)] \cap P$ the second component of $\psi$ is flat with the exception of the grey zone, where the gradient is equal to $(0,1)$.

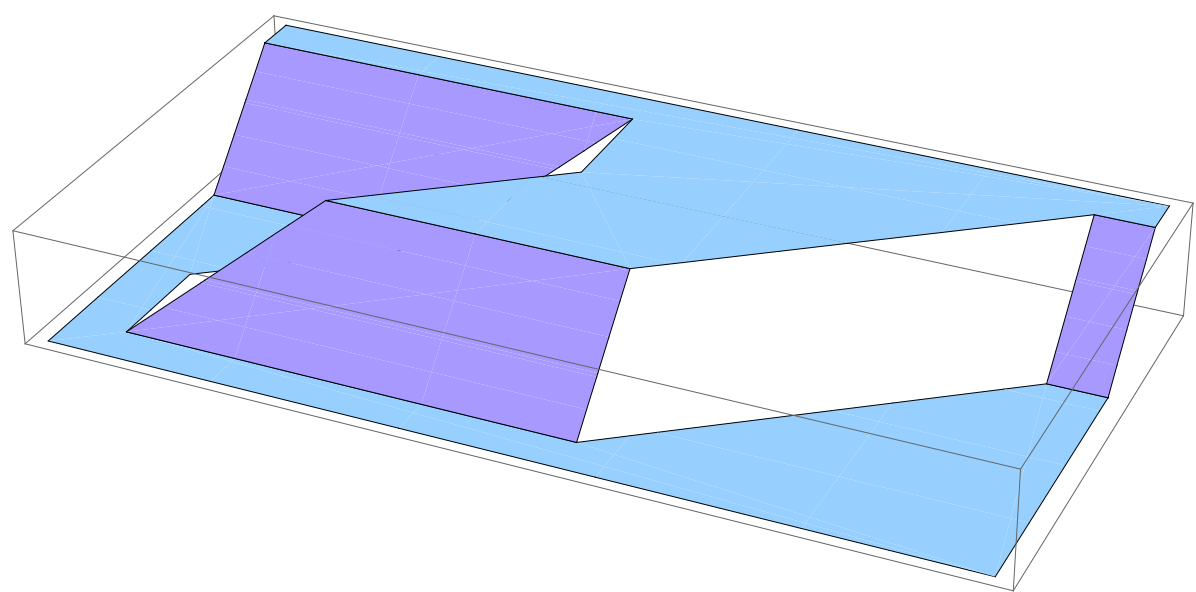

Figure 13. The graph of the second component of $\psi$ in $[(0,2) \times(0,1)] \cap P$.

Step 3. The set $H$ is convex and so the inclusion $(K \cup L)^{q c} \subseteq H$ is immediate.

As a direct consequence of [14, Theorem 1], for any $C \in \mathbb{M}_{\text {sym }}^{2}$ the set

$$
N_{C}:=\left\{D \in \mathbb{M}_{\text {sym }}^{2}: D-C \text { is not positive definite }\right\}
$$

is quasiconvex. Since for any $D \in H \backslash(K \cup L)$ there exists a $C \in H \backslash(K \cup L)$ such that

$$
D \notin N_{C}, \quad \text { whereas } \quad K \cup L \subseteq N_{C},
$$

we can conclude that

$$
(K \cup L)^{q c} \subseteq H \cap\left(\bigcap_{C \in H \backslash(K \cup L)} N_{C}\right)=K \cup L
$$


and then $K \cup L$ is quasiconvex.

Remark 5. The proof of Theorem 6 does not take advantage of the particular structure of $W$ but it is based only on the fact that $W^{-1}(0)=K$. Therefore, instead of $W$ we can consider the function $V: \mathbb{M}^{2} \rightarrow[0,+\infty)$ defined as the quasiconvex envelope of $\operatorname{dist}(\cdot, K)^{p}, p \in(1,+\infty)$. Indeed, since $K$ is polyconvex (because zero levelset of the polyconvex function $W$ ), by Lemma 5 it follows that $V^{-1}(0)=K$. Note that in this way our counterexample covers also the case of energy densities with growth $p \in(1,2)$.

Remark 6. Let $N$ be a convex and compact subset of $\mathbb{M}^{2}$ sufficiently large so that $\nabla \psi \in N$ a.e. in $Q_{2}$, where $\psi$ is defined as in (4.5). Consider now the function $V: \mathbb{R}^{2} \times \mathbb{M}^{2} \rightarrow[0,+\infty)$ defined by

$$
V(x, \Lambda):=\chi_{P}(x) W(\Lambda)+\left(1-\chi_{P}(x)\right) \operatorname{dist}(\Lambda, N)^{p} .
$$

Since $W_{\text {cell }} \leq V_{\text {cell }}$, we have the inclusion $V_{\text {cell }}^{-1}(0) \subseteq K \cup L$. We also have the inclusion $K \cup M \subseteq V_{\text {hom }}^{-1}(0)$ : in fact $K \subseteq V_{\text {hom }}^{-1}(0)$ (because $K \subseteq N$ ) and $\operatorname{diag}\left(h_{1} / 2, h_{3}\right) \in V_{\text {hom }}^{-1}(0)$ (by using again $\psi$ ). In this way we can conclude that also by mixing a polyconvex function and a convex function, the inequality $Q V_{\text {cell }}>V_{\text {hom }}$ can occur.

\section{Appendix: Stefan MülleR's example in Dimension three}

To show that Proposition 1 is not peculiar to dimension two, let us consider the corresponding energy for a three-dimensional soft material reinforced by a strong plate. The energy is now given by $W_{0}: \mathbb{M}^{3} \ni \Lambda \mapsto|\Lambda|^{4}+f(\operatorname{det} \Lambda)$ where

$$
f(z):= \begin{cases}\frac{12(1+a)^{2}}{z+a}-12(1+a)-9 & \text { if } z>0 \\ \frac{12(1+a)^{2}}{a}-12(1+a)-9-\frac{12(1+a)^{2}}{a^{2}} z & \text { if } z \leq 0\end{cases}
$$

for $a \in(0,1 / 2)$. The energy density under consideration is still of the form $W^{\eta}: \mathbb{R}^{3} \times \mathbb{M}^{3} \rightarrow$ $[0,+\infty),(y, \Lambda) \mapsto \chi^{\eta}(y) W_{0}(\Lambda)$ where $\chi^{\eta}$ is the $Q$-periodic extension on $\mathbb{R}^{3}$ of

$$
\chi^{\eta}(y):=\left\{\begin{array}{ll}
1 & \text { if } y_{1} \in(0,1 / 2) \\
\eta & \text { if } y_{1} \in[1 / 2,1)
\end{array},\right.
$$

with $Q=(0,1)^{3} \ni y=\left(y_{1}, y_{2}, y_{3}\right)$ and $\eta>0$. Such an energy density is nonnegative, polyconvex, frame-invariant and its zero-levelset is $\mathrm{SO}_{3}$.

Proposition 2. For all $\lambda_{1}, \lambda_{2} \in(0,1)$, there exists $c>0$ independent of $\eta$ such that

$$
Q W_{\text {cell }}^{\eta}(\Lambda) \leq \eta c,
$$

where $\Lambda:=\operatorname{diag}\left(1, \lambda_{1}, \lambda_{2}\right)$.

Proof. Essentially, one has to construct a Lipschitz domain $U$ of $\mathbb{R}^{3}$, a function $\phi \in$ $W_{\text {per }}^{1, \infty}\left(U, \mathbb{R}^{3}\right)$ and a function $\varphi \in L^{\infty}\left(U, W_{\text {per }}^{1, \infty}\left(Q, \mathbb{R}^{3}\right)\right)$ such that $\Lambda+\nabla \phi(x)+\nabla_{y} \varphi(x, y) \in$ $\mathrm{SO}_{3}$ for all $x \in U$ and $y \in(0,1 / 2) \times(0,1)^{2}$ (the strong phase of the material).

Notation. The canonical basis of $\mathbb{R}^{3}$ is denoted by $\left\{\mathbf{e}_{\mathbf{1}}, \mathbf{e}_{\mathbf{2}}, \mathbf{e}_{\mathbf{3}}\right\}$. 
We will use the angles $\theta$ and $\gamma$ defined by

$$
\begin{aligned}
& \cos \theta=\lambda_{1}, \quad \sin \theta=\sqrt{1-\lambda_{1}^{2}}, \\
& \cos \gamma=\lambda_{2}, \quad \sin \gamma=\sqrt{1-\lambda_{2}^{2}} .
\end{aligned}
$$

We set $\rho:=\sqrt{\sin ^{2} \theta+\cos ^{2} \theta \sin ^{2} \gamma}$ and define the unit vectors $\mathbf{e}_{\mathbf{4}}$ and $\mathbf{e}_{\mathbf{5}}$ by

$$
\begin{aligned}
\mathbf{e}_{4} & :=\frac{1}{\rho}\left(\sin \theta \mathbf{e}_{2}+\cos \theta \sin \gamma \mathbf{e}_{3}\right), \\
\mathbf{e}_{5} & :=\frac{1}{\rho}\left(\sin \theta \mathbf{e}_{2}-\cos \theta \sin \gamma \mathbf{e}_{3}\right) .
\end{aligned}
$$

Definition of $U$. In order to describe $U$, we need the following quantity

$$
\tau:=\frac{\cos \theta \sin \gamma}{\sin \theta}
$$

We set $U:=U_{1} \cup U_{2} \cup U_{3} \cup U_{4}$, where

$$
\begin{aligned}
& U_{1}:=\left\{\left(x_{1}, x_{2}, x_{3}\right): 0<x_{1}<1 ; 0<x_{3} \leq 1 / 2 ; 1 / 2-\tau x_{3} \leq x_{2}<1-\tau x_{3}\right\}, \\
& U_{2}:=\left\{\left(x_{1}, x_{2}, x_{3}\right): 0<x_{1}<1 ; 0<x_{3} \leq 1 / 2 ;-\tau x_{3}<x_{2} \leq 1 / 2-\tau x_{3}\right\}, \\
& U_{3}:=\left\{\left(x_{1}, x_{2}, x_{3}\right): 0<x_{1}<1 ; 1 / 2 \leq x_{3}<1 ; 1 / 2-\tau\left(1-x_{3}\right) \leq x_{2}<1-\tau\left(1-x_{3}\right)\right\}, \\
& U_{4}:=\left\{\left(x_{1}, x_{2}, x_{3}\right): 0<x_{1}<1 ; 1 / 2 \leq x_{3}<1 ;-\tau\left(1-x_{3}\right)<x_{2} \leq 1 / 2-\tau\left(1-x_{3}\right)\right\} .
\end{aligned}
$$

The domains $U_{1}, U_{2}, U_{3}, U_{4}$ are sketched on Figure 14 . Note that the interface between $U_{1}$ and $U_{2}$ (resp. $U_{3}$ and $U_{4}$ ) is perpendicular to $\mathbf{e}_{4}$ (resp. $\mathbf{e}_{5}$ ).

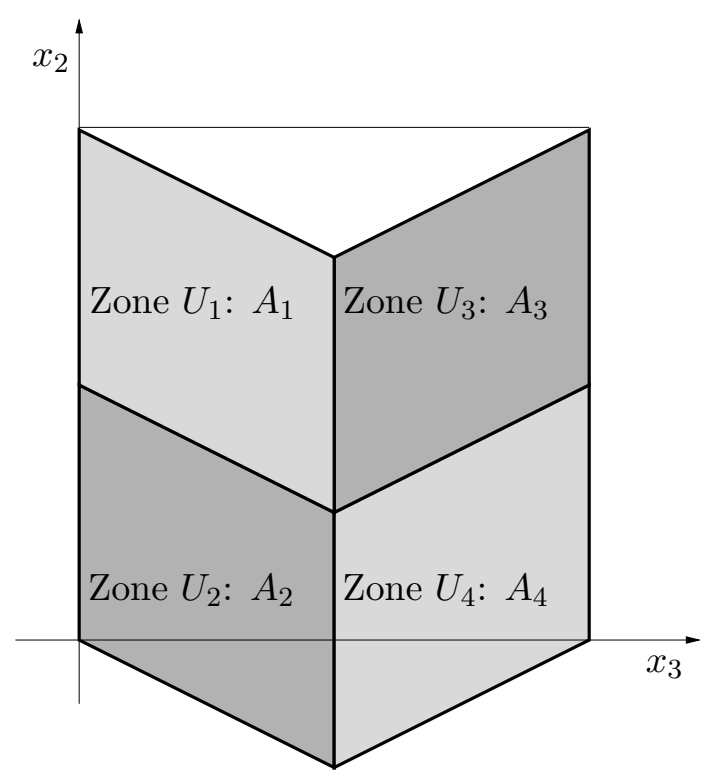

Figure 14. Domain U (in the plane generated by $\left\{\mathbf{e}_{2}, \mathbf{e}_{3}\right\}$ ). 
Definition of $\phi$. We consider the piecewise constant function $G \in L^{\infty}\left(U, \mathbb{M}^{3}\right)$ of the form

$$
G=\left(\begin{array}{ccc}
0 & \times & \times \\
0 & \times & \times \\
0 & 0 & 0
\end{array}\right)
$$

where the non zero two-dimensional submatrix $\left(\begin{array}{cc}x & \times \\ x & \times\end{array}\right)$ is one the following four possible submatrices:

$$
\begin{aligned}
& A_{1}:=\left(\begin{array}{cc}
\sin \theta & \cos \theta \sin \gamma \\
0 & -\sin \theta \sin \gamma
\end{array}\right) \text { in } U_{1}, \quad A_{2}:=\left(\begin{array}{cc}
-\sin \theta & -\cos \theta \sin \gamma \\
0 & -\sin \theta \sin \gamma
\end{array}\right) \text { in } U_{2}, \\
& A_{3}:=\left(\begin{array}{cc}
\sin \theta & -\cos \theta \sin \gamma \\
0 & \sin \theta \sin \gamma
\end{array}\right) \text { in } U_{3}, \quad A_{4}:=\left(\begin{array}{cc}
-\sin \theta & \cos \theta \sin \gamma \\
0 & \sin \theta \sin \gamma
\end{array}\right) \text { in } U_{4},
\end{aligned}
$$

according to Figure 14 .

Let us check that $G$ is a gradient field:

$$
\begin{aligned}
A_{1}-A_{3} & =2\left[\cos \theta \sin \gamma \mathbf{e}_{1}-\sin \theta \sin \gamma \mathbf{e}_{2}\right] \otimes \mathbf{e}_{3} \\
A_{2}-A_{4} & =2\left[-\cos \theta \sin \gamma \mathbf{e}_{1}-\sin \theta \sin \gamma \mathbf{e}_{2}\right] \otimes \mathbf{e}_{3} \\
A_{1}-A_{2} & =2 \mathbf{e}_{1} \otimes\left[\sin \theta \mathbf{e}_{2}+\cos \theta \sin \gamma \mathbf{e}_{3}\right] \\
& =2 \rho \mathbf{e}_{1} \otimes \mathbf{e}_{4} \\
A_{3}-A_{4} & =2 \mathbf{e}_{1} \otimes\left[\sin \theta \mathbf{e}_{2}-\cos \theta \sin \gamma \mathbf{e}_{3}\right] \\
& =2 \rho \mathbf{e}_{1} \otimes \mathbf{e}_{5} .
\end{aligned}
$$

These couples of matrices being rank-one connected, $G$ is actually a gradient field $\nabla \phi$ on $U$. This gradient can be extended by periodicity (due to the boundary conditions).

Definition of $\varphi$. We consider the following specific rotations in 3D, which are compositions of rotations around $\mathbf{e}_{2}$ and $\mathbf{e}_{3}$ :

$$
\begin{aligned}
R(\alpha, \beta): & =\left(\begin{array}{ccc}
\cos \alpha & -\sin \alpha & 0 \\
\sin \alpha & \cos \alpha & 0 \\
0 & 0 & 1
\end{array}\right)\left(\begin{array}{ccc}
\cos \beta & 0 & \sin \beta \\
0 & 1 & 0 \\
-\sin \beta & 0 & \cos \beta
\end{array}\right) \\
& =\left(\begin{array}{ccc}
\cos \beta \cos \alpha & -\sin \alpha & \cos \alpha \sin \beta \\
\cos \beta \sin \alpha & \cos \alpha & \sin \alpha \sin \beta \\
-\sin \beta & 0 & \cos \beta
\end{array}\right),
\end{aligned}
$$

where $\alpha$ and $\beta$ are the two angles. We also denote by $\bar{\chi}$ the $Q$-periodic extension on $\mathbb{R}^{3}$ of

$$
\bar{\chi}(y):=\left\{\begin{array}{ll}
1 & \text { if } y_{1} \in(0,1 / 2) \\
-1 & \text { if } y_{1} \in[1 / 2,1)
\end{array} .\right.
$$

We construct a function $\varphi$ of the form $\varphi(x, y):=\sum_{i=1}^{4} \chi_{U_{i}}(x) \varphi_{i}(y)$, where $\varphi_{1}, \ldots, \varphi_{4}$ are defined as follows.

Zone 1. For $x \in U_{1}$,

$$
\Lambda+\nabla \phi(x)=\left(\begin{array}{ccc}
1 & \sin \theta & \cos \theta \sin \gamma \\
0 & \cos \theta & -\sin \theta \sin \gamma \\
0 & 0 & \cos \gamma
\end{array}\right)
$$


We then choose $\varphi_{1} \in W_{\text {per }}^{1, \infty}\left(Q, \mathbb{R}^{3}\right)$ such that

$$
\nabla \varphi_{1}(y)=\bar{\chi}(y)\left(\begin{array}{ccc}
\cos \gamma \cos \theta-1 & 0 & 0 \\
-\cos \gamma \sin \theta & 0 & 0 \\
-\sin \gamma & 0 & 0
\end{array}\right) .
$$

In the strong phase $(\bar{\chi}(y)=1)$,

$$
\Lambda+\nabla \phi(x)+\nabla \varphi_{1}(y)=\left(\begin{array}{ccc}
\cos \gamma \cos \theta & \sin \theta & \cos \theta \sin \gamma \\
-\cos \gamma \sin \theta & \cos \theta & -\sin \theta \sin \gamma \\
-\sin \gamma & 0 & \cos \gamma
\end{array}\right)=R(\widetilde{\theta}, \gamma)
$$

is a rotation, with $\widetilde{\theta}=-\theta$. In the soft phase $(\bar{\chi}(y)=-1)$

$$
\Lambda+\nabla \phi(x)+\nabla \varphi_{1}(y)=\left(\begin{array}{ccc}
2-\cos \gamma \cos \widetilde{\theta} & -\sin \widetilde{\theta} & \cos \widetilde{\theta} \sin \gamma \\
-\cos \gamma \sin \widetilde{\theta} & \cos \widetilde{\theta} & \sin \tilde{\theta} \sin \gamma \\
\sin \gamma & 0 & \cos \gamma
\end{array}\right) .
$$

Zone 2. For $x \in U_{2}$,

$$
\Lambda+\nabla \phi(x)=\left(\begin{array}{ccc}
1 & -\sin \theta & -\cos \theta \sin \gamma \\
0 & \cos \theta & -\sin \theta \sin \gamma \\
0 & 0 & \cos \gamma
\end{array}\right)
$$

We then choose $\varphi_{2} \in W_{\text {per }}^{1, \infty}\left(Q, \mathbb{R}^{3}\right)$ such that

$$
\nabla \varphi_{2}(y)=\bar{\chi}(y)\left(\begin{array}{ccc}
\cos \gamma \cos \theta-1 & 0 & 0 \\
\cos \gamma \sin \theta & 0 & 0 \\
\sin \gamma & 0 & 0
\end{array}\right)
$$

In the strong phase $(\bar{\chi}(y)=1)$,

$$
\Lambda+\nabla \phi(x)+\nabla \varphi_{2}(y)=\left(\begin{array}{ccc}
\cos \gamma \cos \theta & -\sin \theta & -\cos \theta \sin \gamma \\
\cos \gamma \sin \theta & \cos \theta & -\sin \theta \sin \gamma \\
\sin \gamma & 0 & \cos \gamma
\end{array}\right)=R(\theta, \widetilde{\gamma})
$$

is a rotation, with $\widetilde{\gamma}=-\gamma$. In the soft phase $(\bar{\chi}(y)=-1)$,

$$
\Lambda+\nabla \phi(x)+\nabla \varphi_{2}(y)=\left(\begin{array}{ccc}
2-\cos \widetilde{\gamma} \cos \theta & -\sin \theta & \cos \theta \sin \widetilde{\gamma} \\
-\cos \widetilde{\gamma} \sin \theta & \cos \theta & \sin \theta \sin \widetilde{\gamma} \\
\sin \widetilde{\gamma} & 0 & \cos \widetilde{\gamma}
\end{array}\right) .
$$

Zones 3 and 4. Proceeding as above, one may find $\varphi_{3}, \varphi_{4} \in W_{\text {per }}^{1, \infty}\left(Q, \mathbb{R}^{3}\right)$ such that for all $x \in U_{3}$ and $y \in(0,1 / 2) \times(0,1)^{2}$,

$$
\Lambda+\nabla \phi(x)+\nabla \varphi_{3}(y)=\left(\begin{array}{ccc}
\cos \gamma \cos \theta & \sin \theta & -\cos \theta \sin \gamma \\
-\cos \gamma \sin \theta & \cos \theta & \sin \theta \sin \gamma \\
\sin \gamma & 0 & \cos \gamma
\end{array}\right)=R(\widetilde{\theta}, \widetilde{\gamma})
$$

is a rotation, with $\widetilde{\theta}=-\theta, \widetilde{\gamma}=-\gamma$; and for all $x \in U_{4}$ and $y \in(0,1 / 2) \times(0,1)^{2}$,

$$
\Lambda+\nabla \phi(x)+\nabla \varphi_{4}(y)=\left(\begin{array}{ccc}
\cos \gamma \cos \theta & -\sin \theta & \cos \theta \sin \gamma \\
\cos \gamma \sin \theta & \cos \theta & \sin \theta \sin \gamma \\
-\sin \gamma & 0 & \cos \gamma
\end{array}\right)=R(\theta, \gamma)
$$


is a rotation.

Finally, we are in position to conclude the proof. By using $\phi$ and $\varphi$ defined above as test-functions, one obtains

$$
Q W_{\text {cell }}^{\eta}(\Lambda) \leq \int_{U} \int_{Q} W^{\eta}\left(y, \Lambda+\nabla \phi(x)+\nabla_{y} \varphi(x, y)\right) d y d x \leq \eta c,
$$

where $c=\max \left(W\left(C_{i}\right)\right) / 2$ and $\left\{C_{i}\right\}_{i}$ is the finite set of values taken by $\Lambda+\nabla \phi(x)+$ $\nabla_{y} \varphi(x, y)$ in the soft phase.

\section{ACKNOWLEDGMENTS}

M. Barchiesi wishes to thank Andrea Braides, Giovanni Leoni and Enzo Nesi for their useful comments and suggestions. A. Gloria gratefully thanks Andrea Braides and Stefan Müller for stimulating discussions. The research of M. Barchiesi was supported by the Center for Nonlinear Analysis (NSF Grants No. DMS-0405343 and DMS-0635983). The research of A. Gloria was supported by the Hausdorff Center for Mathematics. He also acknowledges the support from the Marie Curie Research Training Network MRTNCT-2004-505226 'Multi-scale modelling and characterisation for phase transformations in advanced materials' (MULTIMAT).

\section{REFERENCES}

[1] R. Alicandro and M. Cicalese. A general integral representation result for the continuum limits of discrete energies with superlinear growth. SIAM J. Math. Anal., 36:1-37, 2004.

[2] R. Alicandro, M. Cicalese, and A. Gloria. Integral representation results for energies defined on stochastic lattices and application to nonlinear elasticity. In preparation.

[3] J.-F. Babadjian and M. Barchiesi. A variational approach to the local character of G-closure: the convex case. Annales de l'institut Henri Poincaré: Analyse non linéaire, 2008. To appear.

[4] J.M. Ball and R.D. James. Fine mixtures as minimizers of energy. Arch. Rat. Mech. Anal., 100:13-52, 1987.

[5] J. M. Ball \& R. D. James. Proposed experimental tests of a theory of fine microstructure and the two-well problem. Phil. Trans. Roy. Soc. London A, 338:389-450, 1992.

[6] M. Barchiesi. Loss of polyconvexity by homogenization: a new example. Calc. Var. Partial Differential Equations, 30:215-230, 2007.

[7] A. Braides and A. Defranceschi. Homogenization of Multiple Integrals, volume 12 of Oxford Lecture Series in Mathematics and Its Applications. Oxford University Press, 1998.

[8] B. Dacorogna. Direct methods in the calculus of variations, vol. 78 of Applied Mathematical Sciences. Springer, Berlin, 2nd ed., 2008.

[9] I. Fonseca \& G. Leoni. Modern methods in the Calculus of Variations: Sobolev spaces. In preparation.

[10] E. Giusti. Direct methods in the calculus of variations. World Scientific Publishing Co. Inc., River Edge, NJ, 2003.

[11] T. Iwaniec, G. Verchota \& A. Vogel. The Failure of Rank-One Connections. Arch. Rational Mech. Anal., 163:125-169, 2002.

[12] S. Müller. Homogenization of nonconvex integral functionals and cellular elastic materials. Arch. Rat. Anal. Mech., 99:189-212, 1987.

[13] S. Müller. Variational models for microstructure and phase transitions. In Calculus of variations and geometric evolution problems (Cetraro, 1996), volume 1713 of Lecture Notes in Math., pages 85-210. Springer, Berlin, 1999.

[14] V. Šverák. New examples of quasiconvex functions. Arch. Rational Mech. Anal., 119:293-300, 1992.

[15] V. Šverák. On the problem of two wells. volume 54 of IMA Vol. Appl. Math., pages 183-189. Springer, 1993. 
(Marco Barchiesi) CNA, Carnegie Mellon University, Pittsburgh, PA 15213, USA

E-mail address: marcob@andrew. cmu.edu

(Antoine Gloria) IAM, Universität Bonn, Wegelerstr. 10, 53115 Bonn, Germany

E-mail address: gloria@iam.uni-bonn.de 